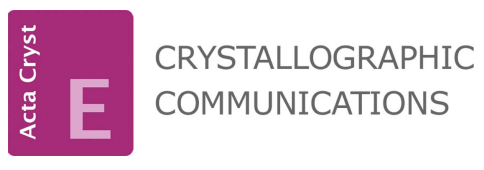

ISSN 2056-9890

Received 2 April 2021

Accepted 4 April 2021

Edited by W. T. A. Harrison, University of Aberdeen, Scotland

Keywords: heterocyclic compounds; benzo[d]thiazoles; crystal structure; molecular conformation; disorder; hydrogen bonding; halogen bonding.

CCDC references: 2075271; 2075270; 2075269

Supporting information: this article has supporting information at journals.iucr.org/e

\section{Different patterns of supramolecular aggregation in three amides containing $N$-(benzo[d]thiazolyl) substituents}

\author{
Ninganayaka Mahesha, ${ }^{a}$ Hemmige S. Yathirajan, ${ }^{a *}$ Holalagudu A. Nagma Banu, ${ }^{b}$ \\ Balakrishna Kalluraya, ${ }^{b}$ Sabine Foro ${ }^{c}$ and Christopher Glidewell ${ }^{d}$
}

\begin{abstract}
${ }^{\mathbf{a}}$ Department of Studies in Chemistry, University of Mysore, Manasagangotri, Mysuru-570 006, India, ${ }^{\mathbf{b}}$ Department of Studies in Chemistry, Mangalore University, Mangalagangotri, Mangalore-574199, India, ' Institute of Materials Science, Darmstadt University of Technology, Alarich-Weiss-Strasse 2, D-64287 Darmstadt, Germany, and ${ }^{\mathbf{d}}$ School of Chemistry, University of St Andrews, St Andrews, Fife KY16 9ST, UK. *Correspondence e-mail: yathirajan@hotmail.com
\end{abstract}

Crystal structures are reported for three amides containing $N$-benzo $[d]$ thiazole substituents. In $N$-(benzo[ $d]$ thiazol-6-yl)-3-bromobenzamide, $\mathrm{C}_{14} \mathrm{H}_{9} \mathrm{BrN}_{2} \mathrm{OS}$, where the two ring systems are nearly parallel to one another [dihedral angle $\left.=5.8(2)^{\circ}\right]$, the molecules are linked by $\mathrm{N}-\mathrm{H} \cdots \mathrm{O}$ and $\mathrm{C}-\mathrm{H} \cdots \mathrm{N}$ hydrogen bonds to form ribbons of $R_{3}^{3}(19)$ rings, which are linked into sheets by short $\mathrm{Br} \cdots \mathrm{Br}$ interactions [3.5812 (6) $\AA$ ].$N$-(6-Methoxybenzo[ $d$ ] thiazol-2-yl)-2-nitrobenzamide, $\mathrm{C}_{15} \mathrm{H}_{11} \mathrm{~N}_{3} \mathrm{O}_{4} \mathrm{~S}$, crystallizes with $Z^{\prime}=2$ in space group Pna2 ${ }_{1}$ : the dihedral angles between the ring systems [46.43(15) and $\left.66.35(13)^{\circ}\right]$ are significantly different in the independent molecules and a combination of two $\mathrm{N}-\mathrm{H} \cdots \mathrm{N}$ and five $\mathrm{C}-\mathrm{H} \cdots \mathrm{O}$ hydrogen bonds links the molecules into a threedimensional network. The molecules of 5-cyclopropyl- $N$-(6-methoxybenzo[d]thiazol-2-yl)isoxazole-3-carboxamide, $\mathrm{C}_{15} \mathrm{H}_{13} \mathrm{~N}_{3} \mathrm{O}_{3} \mathrm{~S}$, exhibit two forms of disorder, in the methoxy group and in the cyclopropylisoxazole unit; symmetryrelated pairs of molecules are linked into dimers by pairwise $\mathrm{N}-\mathrm{H} \cdots \mathrm{N}$ hydrogen bonds. Comparisons are made with the structures of some related compounds.

\section{Chemical context}

Compounds containing the benzo[ $[d]$ thiazole unit exhibit a wide range of biological and medicinal activities, which have been reviewed by Henary et al. (2013). Notable examples include the presence of the benzo[d]thiazole nucleus in firefly luciferin, (4S)-2-(6-hydroxybenzo[d]thiazol-2-yl)-4,5-dihydrothiazole-4-carboxylic acid (White et al., 1963), action as potent and selective human adenosine $\mathrm{A}_{3}$ receptor antagonists (Jung et al., 2004) and cholinesterase inhibitors (Imramovský et al., 2013). In addition, applications in Green Chemistry have very recently been reviewed (Gao et al., 2020).

Against this diverse background, we report here the synthesis and structures of three carboxamides containing the benzo $[d]$ thiazole nucleus, namely: $N$-(benzo $[d]$ thiazol-6-yl)-3bromobenzamide (I), $N$-(6-methoxybenzo[ $d]$ thiazol-2-yl)-2nitrobenzamide (II) and $N$-(6-methoxybenzo[d]thiazol-2-yl)5-cyclopropylisoxazole-3-carboxamide (III). Compounds (I)(III) were prepared in yields exceeding $85 \%$ by the reaction of an amino-substituted benzo $[d]$ thiazole with an acid chloride in the presence of triethylamine. 
<smiles>[10BH2]</smiles>

(I)<smiles>COc1ccc2nc(NC(=O)c3ccccc3[N+](=O)[O-])sc2c1</smiles>

(II)<smiles>COc1ccc2nc(NC(=O)c3cc(C4CC4)on3)sc2c1</smiles>

(III)

\section{Structural commentary}

In compound (I), the amide unit occupies position 6 of the benzo $[d]$ thiazole unit, whereas in compounds (II) and (III), the amide unit is linked to the bicyclic system at position 2. In (I), (Fig. 1) the thiazole ring and the brominated aryl ring are almost parallel, with a dihedral angle between them of

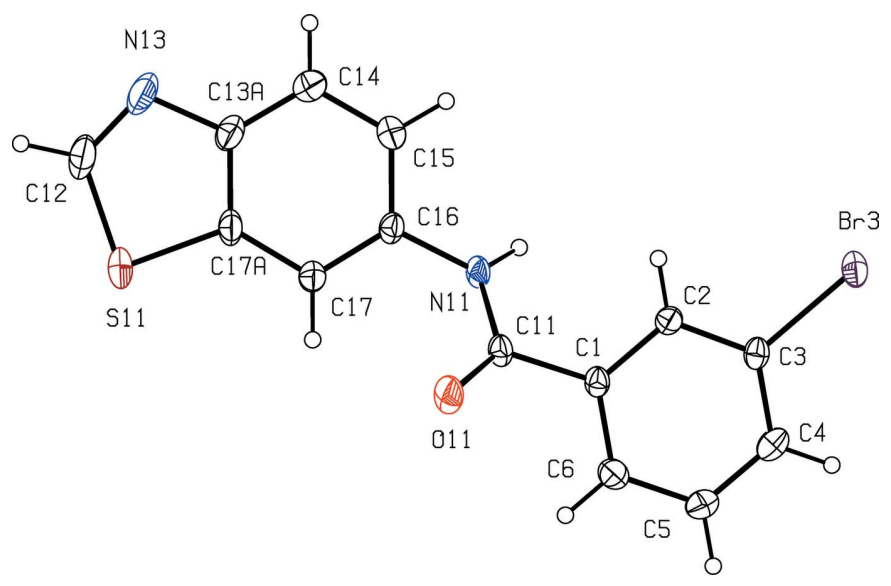

Figure 1

The molecular structure of (I), showing the atom-labelling scheme. Displacement ellipsoids are drawn at the $30 \%$ probability level.

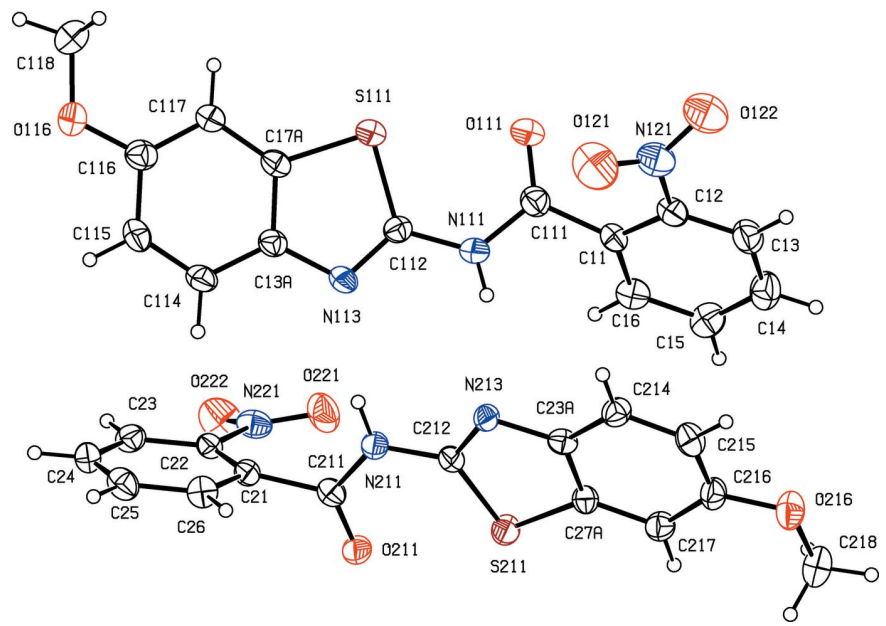

Figure 2

The structures of the two independent molecules in (II), showing the atom-labelling scheme. Displacement ellipsoids are drawn at the $30 \%$ probability level.

$5.8(2)^{\circ}$. However, these rings are not coplanar, as both ring systems in compound (I) are twisted out of the plane of the central amide spacer unit.

Compound (II) crystallizes with $Z^{\prime}=2$, but a search for possible additional crystallographic symmetry revealed none. The different conformations of the two independent molecules (Fig. 2) confirm the absence of additional symmetry. For example, the dihedral angle between the thiazole ring and the nitrated phenyl ring is $46.43(15)^{\circ}$ in molecule 1 containing atom S111, but $66.35(13)^{\circ}$ in molecule 2 containing atom S211. Similarly, the dihedral angles between the nitro groups and the adjacent aryl rings are 34.5 (2) and 17.9 (2) $)^{\circ}$ in molecules 1 and 2, respectively.

The molecule of compound (III) exhibits two forms of disorder. The cyclopropylisoxazole unit is disordered over two sets of atomic sites, with occupancies 0.549 (5) and 0.451 (5), where the two orientations of the isoxazole ring are approximately related by small rotations about the $\mathrm{N}-\mathrm{C}$ and $\mathrm{C}-\mathrm{C}$ bonds involving atom $\mathrm{C} 31$ (Fig. 3). Of more interest is the disorder of the methoxy groups, where the site occupancies are constrained by short non-bonded contacts with adjacent

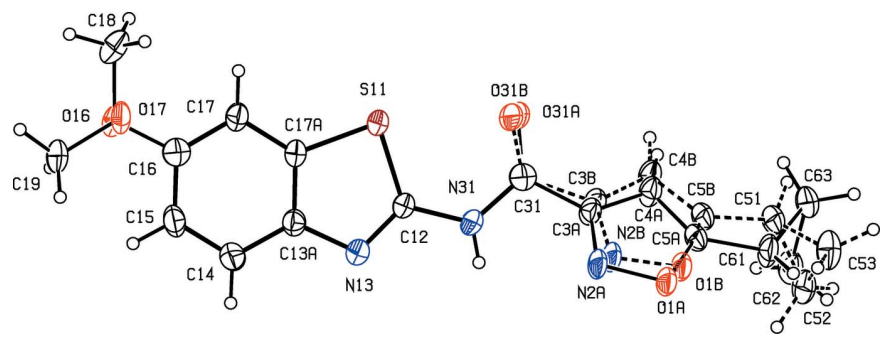

Figure 3

The molecular structure of (III), showing the atom-labelling scheme and the disorder of the cyclopropylisoxazole fragment, where the major disorder component, with occupancy 0.549 (5), is drawn using full lines and the minor disorder component of this fragment, with occupancy 0.451 (5), is drawn using broken lines. The atomic sites O16, O17, C18 and $\mathrm{C} 19$ and the associated $\mathrm{H}$ atoms all have occupancy 0.5 (see Section 2). Displacement ellipsoids are drawn at the $30 \%$ probability level. 
Table 1

Hydrogen bonds and short intermolecular $\operatorname{contacts}\left(\AA{ }^{\circ}\right)$.

$C g 1$ represents the centroid of the ring $\mathrm{C} 13 A / \mathrm{C} 17 A / \mathrm{C} 117 / \mathrm{C} 116 / \mathrm{C} 115 / \mathrm{C} 114$

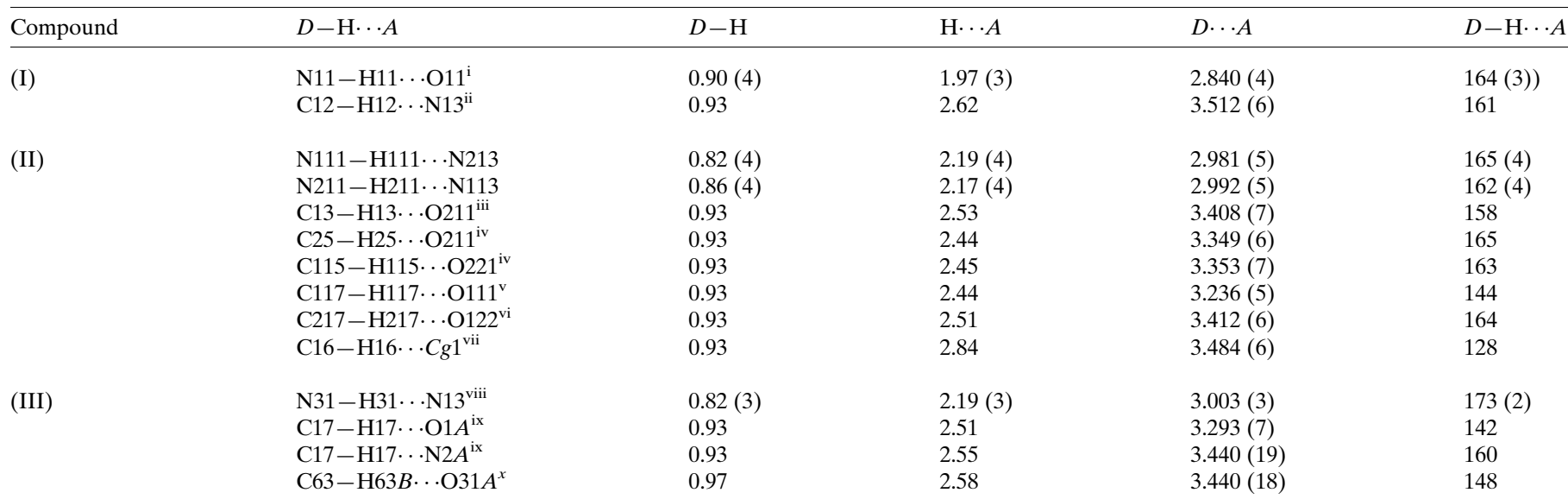

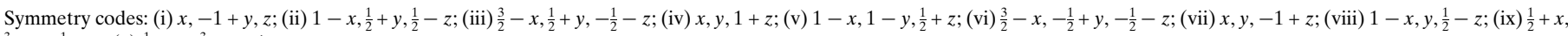
$\frac{3}{2}-y, \frac{1}{2}+z ;(x) \frac{1}{2}-x, \frac{3}{2}-y, 1-z$.

molecules. Thus, the atomic site $\mathrm{C} 18$ in the molecule at $(x, y, z)$ is only 1.840 (8) $\AA$ from the corresponding site in the molecule at $(2-x, y, 1.5-z)$ : hence, only one of these sites can be occupied and this, in turn, limits this site occupancy in each molecule to a maximum value of 0.5 . Similarly, the atomic site C19 at $(x, y, z)$ is only 1.921 (9) $\AA$ from the corresponding site in the molecule at $(2-x, 1-y, 1-z)$, again limiting the site occupancy to a maximum value of 0.5 . Hence the site occupancy for each orientation of the methoxy group must each be exactly 0.5 .

In each of the independent methoxy groups in compound (II), and for each orientation of the methoxy group in

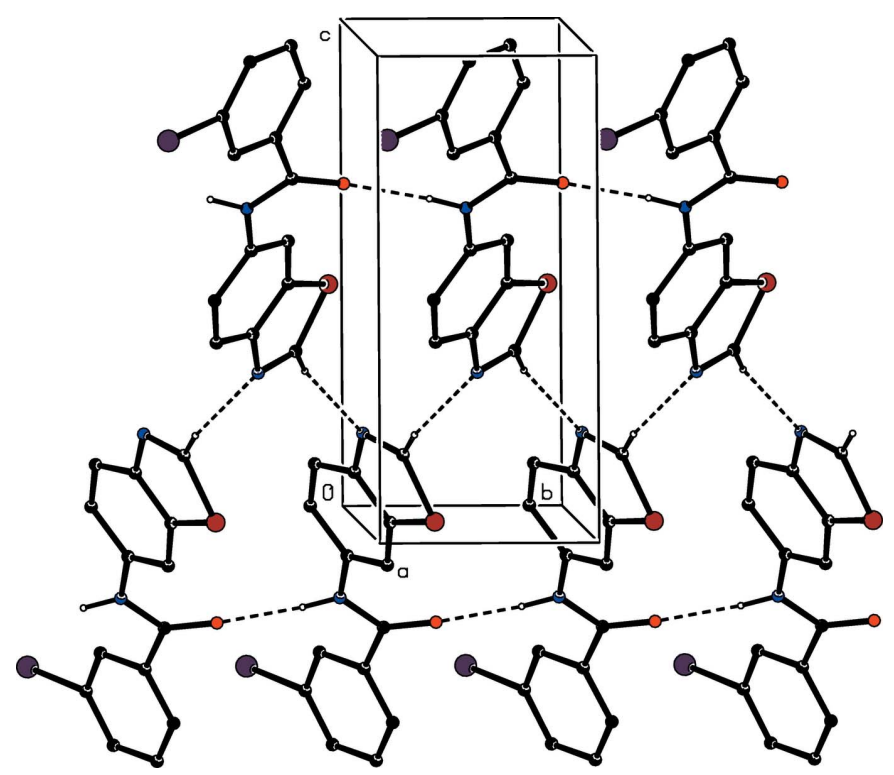

Figure 4

Part of the crystal structure of (I) showing the formation of a ribbon of $R_{3}^{3}(19)$ rings running parallel to [010] and built from $\mathrm{N}-\mathrm{H} \cdots \mathrm{O}$ and $\mathrm{C}-$ $\mathrm{H} \cdots \mathrm{N}$ hydrogen bonds. Hydrogen bonds are drawn as dashed lines and, for the sake of clarity, the $\mathrm{H}$ atoms not involved in the motifs shown have been omitted. compound (III), the two exocyclic $\mathrm{C}-\mathrm{C}-\mathrm{O}$ angles differ by ca $10 \%$, as is generally found in planar, or nearly planar, alkoxyarenes (Seip \& Seip, 1973; Ferguson et al., 1996). In compounds (II) and (III), the maximum displacement of any methoxy $\mathrm{C}$ atoms from the plane of the adjacent aryl ring is 0.144 (9) $\AA$ for atom C218 in compound (II).

\section{Supramolecular features}

The supramolecular assembly of compound (I) is built up from $\mathrm{N}-\mathrm{H} \cdots \mathrm{O}$ and $\mathrm{C}-\mathrm{H} \cdots \mathrm{N}$ hydrogen bonds (Table 1). Molecules related by translation are linked by $\mathrm{N}-\mathrm{H} \cdots \mathrm{O}$ hydrogen bonds to form a C(4) (Etter, 1990; Etter et al., 1990; Bernstein et al., 1995) chain, of the type very commonly found in simple amides (Fun et al., 2011a,b; Praveen et al., 2011; Fun,

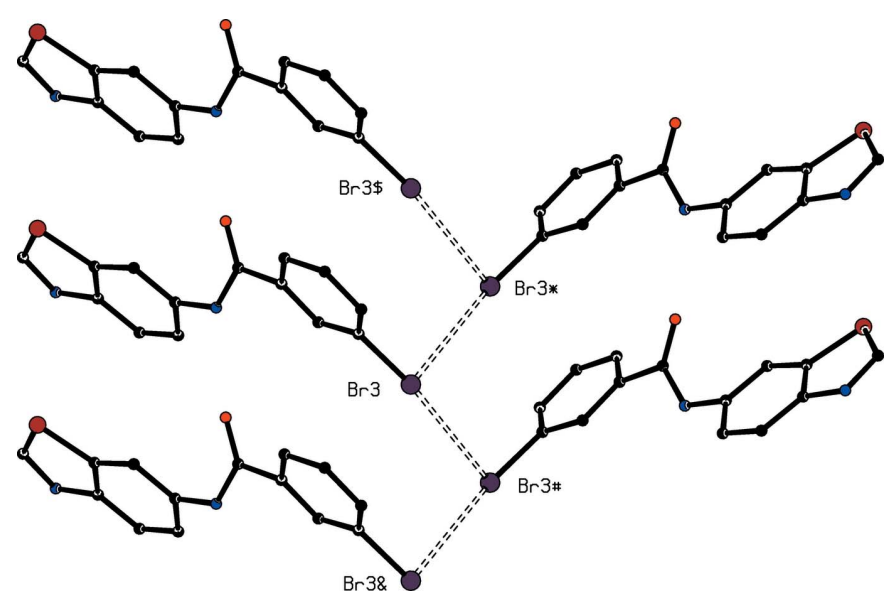

Figure 5

Part of the crystal structure of (I), showing a chain along ([010] containing two independent $\mathrm{Br} \cdots \mathrm{Br}$ interactions (shown as dashed lines). For the sake of clarity, the $\mathrm{H}$ atoms and the unit-cell outline have been omitted. The $\mathrm{Br}$ atoms marked with an asterisk (*), a hash (\#), a dollar sign (\$) or an ampersand $(\&)$ are at the symmetry positions $\left(2-x, \frac{1}{2}+y, \frac{3}{2}-z\right)$, $\left(2-x,-\frac{1}{2}+y, \frac{3}{2}-z\right),(x, 1+y, z)$ and $(x,-1+y, z)$, respectively. 
Quah et al., 2012; Fun, Shahani et al., 2012; Praveen et al., 2013a,b; Nayak et al., 2014): in (I), this chain runs parallel to the [010] direction (Fig. 4). In addition, molecules that are related by the $2_{1}$ screw axis along $(0.5, y, 0.25)$ are linked by $\mathrm{C}-\mathrm{H} \cdots \mathrm{N}$ hydrogen bonds to form a $C(6)$ chain, also running parallel to the [010] direction. The combination of these two chain motifs generates a ribbon of $R_{3}^{3}(19)$ rings along [010] (Fig. 4). Also running through the unit cell is a second ribbon of this type, related to the first by inversion, and containing molecules that are related by the $2_{1}$ screw axis along $(0.5, y$, $0.75)$. Also present in the structure of compound (I) are two intermolecular $\mathrm{Br} \cdots \mathrm{Br}$ contacts that are shorter than the van der Waals radii sum of $3.74 \AA$ (Rowland \& Taylor, 1996). Atom $\mathrm{Br} 3$ in the molecule at $(x, y, z)$ makes contacts with the corresponding atoms at $(2-x, 0.5+y, 1.5-z)$ and $(2-x$, $-0.5+y, 1.5-z)$, with $\mathrm{Br} \cdots \mathrm{Br}$ distances of 3.5812 (6) $\AA$ in

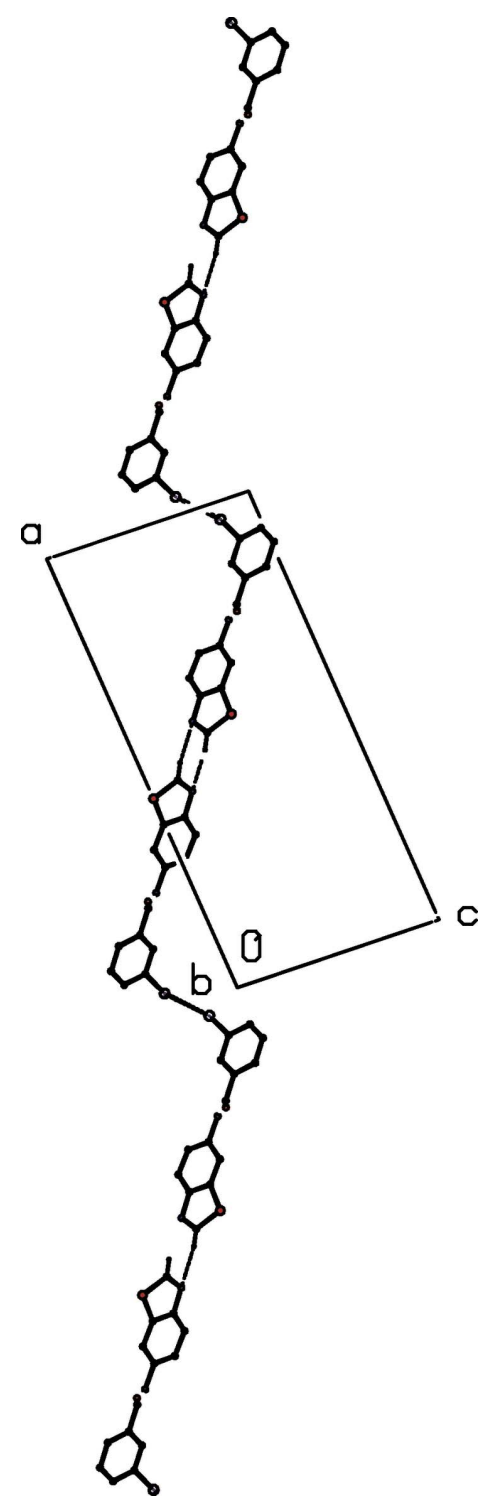

Figure 6

A projection along [010] of part of the crystal structure of (I) showing how the $\mathrm{Br} \cdots \mathrm{Br}$ interactions (dashed lines) link the hydrogen-bonded ribbons into sheets lying parallel to $(10 \overline{1})$. each case; however, the $\mathrm{C}-\mathrm{Br} \cdots \mathrm{Br}$ angles are 92.64 (18) and $166.44(10)^{\circ}$, respectively (Fig. 5), which are consistent with the angular preferences found for such contacts from database analyses (Ramasubbu et al., 1986). The effects of these halogen bonds (Cavallo et al., 2016) are twofold: firstly to generate a chain running parallel to the [010] direction (Fig. 5) and thence to link the hydrogen-bonded ribbons into sheets

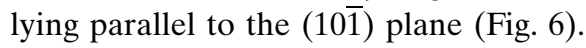

The two independent molecules of compound (II) are linked by two $\mathrm{N}-\mathrm{H} \cdots \mathrm{N}$ hydrogen bonds and five $\mathrm{C}-\mathrm{H} \cdots \mathrm{O}$ hydrogen bonds (Table 1 ), but the $\mathrm{N}-\mathrm{H} \cdots \mathrm{O}$ hydrogen bonds typical of amides are absent. The hydrogen bonds generate a three-dimensional network, whose formation can readily be analysed in terms of a number of simple sub-structures (Ferguson et al., 1998a,b; Gregson et al., 2000). In the simplest of the sub-structures, the two $\mathrm{N}-\mathrm{H} \cdots \mathrm{N}$ hydrogen bonds link the molecules within the selected asymmetric unit to form a dimer, and the other sub-structures follow the different ways in which these dimers can be linked. The $\mathrm{C}-\mathrm{H} \cdots \mathrm{O}$ hydrogen bonds involving atoms $\mathrm{C} 25$ and $\mathrm{C} 115$ link the dimers into a chain of alternating $R_{2}^{2}(8) R_{3}^{3}(18)$ rings running parallel to the [001] direction (Fig. 7); this chain is weakly reinforced by a $\mathrm{C}-\mathrm{H} \cdots \pi$ (arene) interaction (Table 1$)$. In the third substructure, the $\mathrm{C}-\mathrm{H} \cdots \mathrm{O}$ hydrogen bonds involving atoms $\mathrm{C} 13$ and $\mathrm{C} 217$ link the dimers into a chain of rings containing $C_{4}^{4}(24)$ chains and running parallel to the [010] direction (Fig. 8). The combination of the chains along [010] and [001]

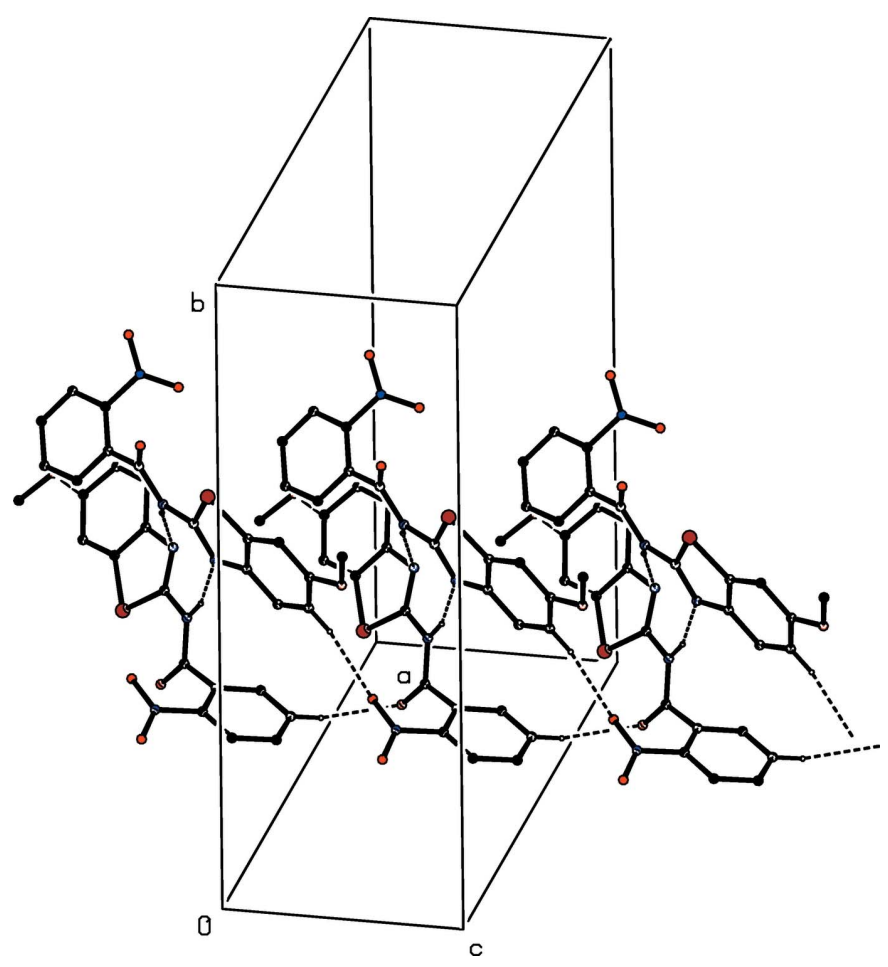

Figure 7

Part of the crystal structure of (II) showing the formation of a chain of $R_{2}^{2}(8)$ and $R_{3}^{3}(18)$ rings running parallel to [001] and built from $\mathrm{N}-\mathrm{H} \cdots \mathrm{N}$ and $\mathrm{C}-\mathrm{H} \cdots \mathrm{O}$ hydrogen bonds. Hydrogen bonds are drawn as dashed lines and, for the sake of clarity, the $\mathrm{H}$ atoms not involved in the motifs shown have been omitted. 


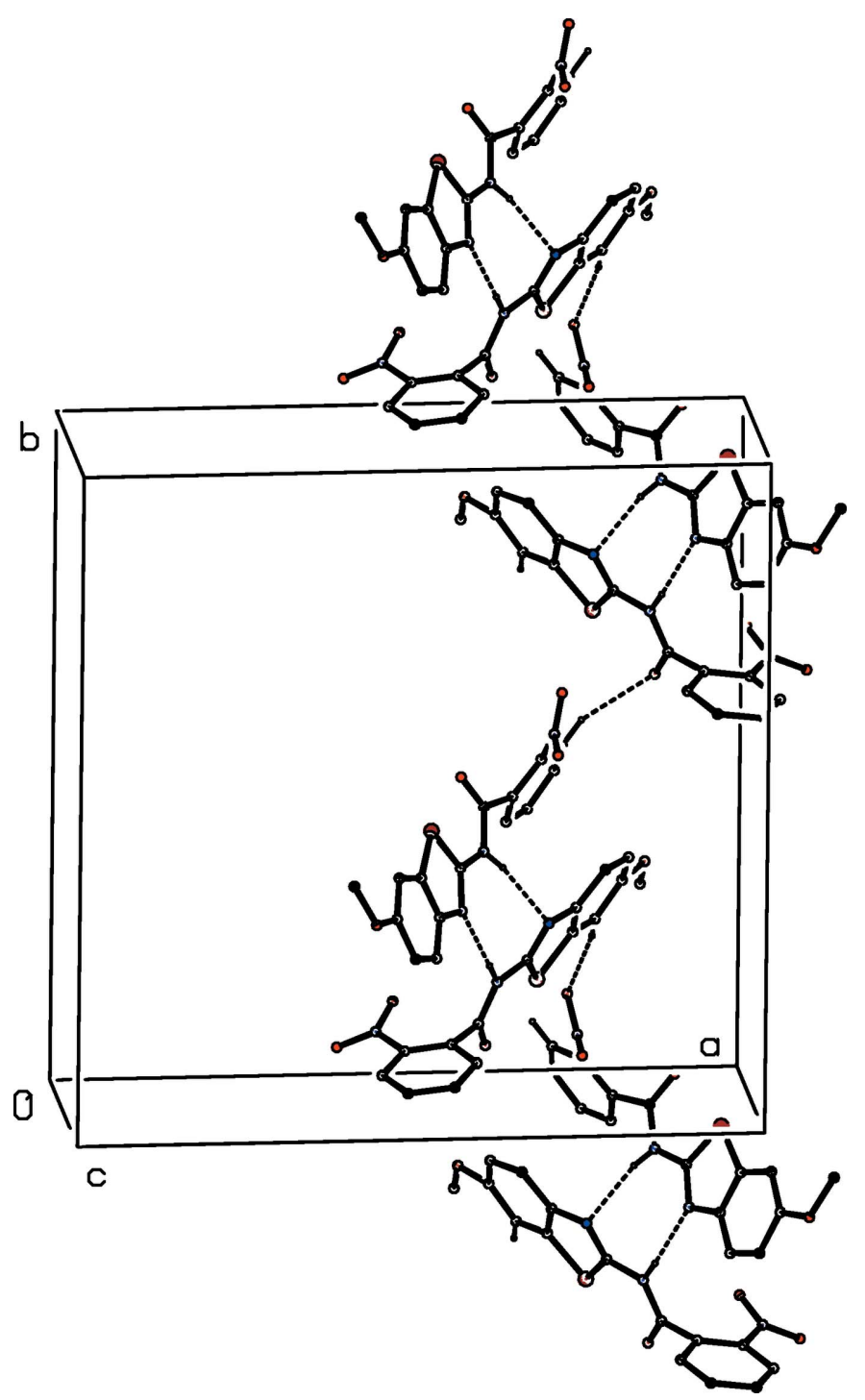

Figure 8

Part of the crystal structure of (II) showing the formation of a chain of rings running parallel to [010] and built from $\mathrm{N}-\mathrm{H} \cdots \mathrm{N}$ and $\mathrm{C}-\mathrm{H} \cdots \mathrm{O}$ hydrogen bonds. Hydrogen bonds are drawn as dashed lines and, for the sake of clarity, the $\mathrm{H}$ atoms not involved in the motifs shown have been omitted.

generates a sheet lying parallel to (100) in the domain $0.5<x<$ 1.0. A second sheet of the type, related to the first by the $2_{1}$ screw axes, lies in the domain $0<x<0.5$, and sheets of this type are linked by the $\mathrm{C}-\mathrm{H} \cdots \mathrm{O}$ hydrogen bond in involving atom $\mathrm{C} 117$, so forming a three-dimensional network: indeed, it is possible to identify a complex chain running parallel to the [100] direction, which defines the linkage of the (100) sheets (Fig. 9).

Analysis of the supramolecular aggregation in compound (III) is complicated by the disorder of the isoxazole ring, since atoms $\mathrm{O} 1 A$ and $\mathrm{N} 2 A$ in the major disorder form act as hydrogen bond acceptors, but atoms $\mathrm{O} 1 B$ and $\mathrm{N} 2 B$ in the minor disorder form do not. As in (II), the $\mathrm{N}-\mathrm{H} \cdots \mathrm{O}$ hydrogen bonds typical of amides are absent from the structure of (III). Molecules of (III) that are related by a twofold rotation axis are linked into cyclic $R_{2}^{2}(8)$ dimers. There is also present an asymmetric three-centre $\mathrm{C}-\mathrm{H} \cdots(\mathrm{N}, \mathrm{O})$ system having atoms $\mathrm{O} 1 A$ and $\mathrm{N} 2 A$ as the acceptors: if these sites had full occupancy, this interaction would generate a chain of rings running parallel to the [101] direction (Fig. 10). However, because of the disorder, this chain is punctuated rather than continuous.

\section{Database survey}

$N$-(Benzo[d]thiazol-2-yl)-3-bromobenzamide (IV) [CSD (Groom et al., 2016) refcode SUQTAC; Odame et al., 2020] is a positional isomer of compound (I), with the amide substituent as position 2 of the benzothiazole unit, rather than at position 6 as in (I). In contrast to compound (I), but consistent with compounds (II) and (III), where the amide units are also

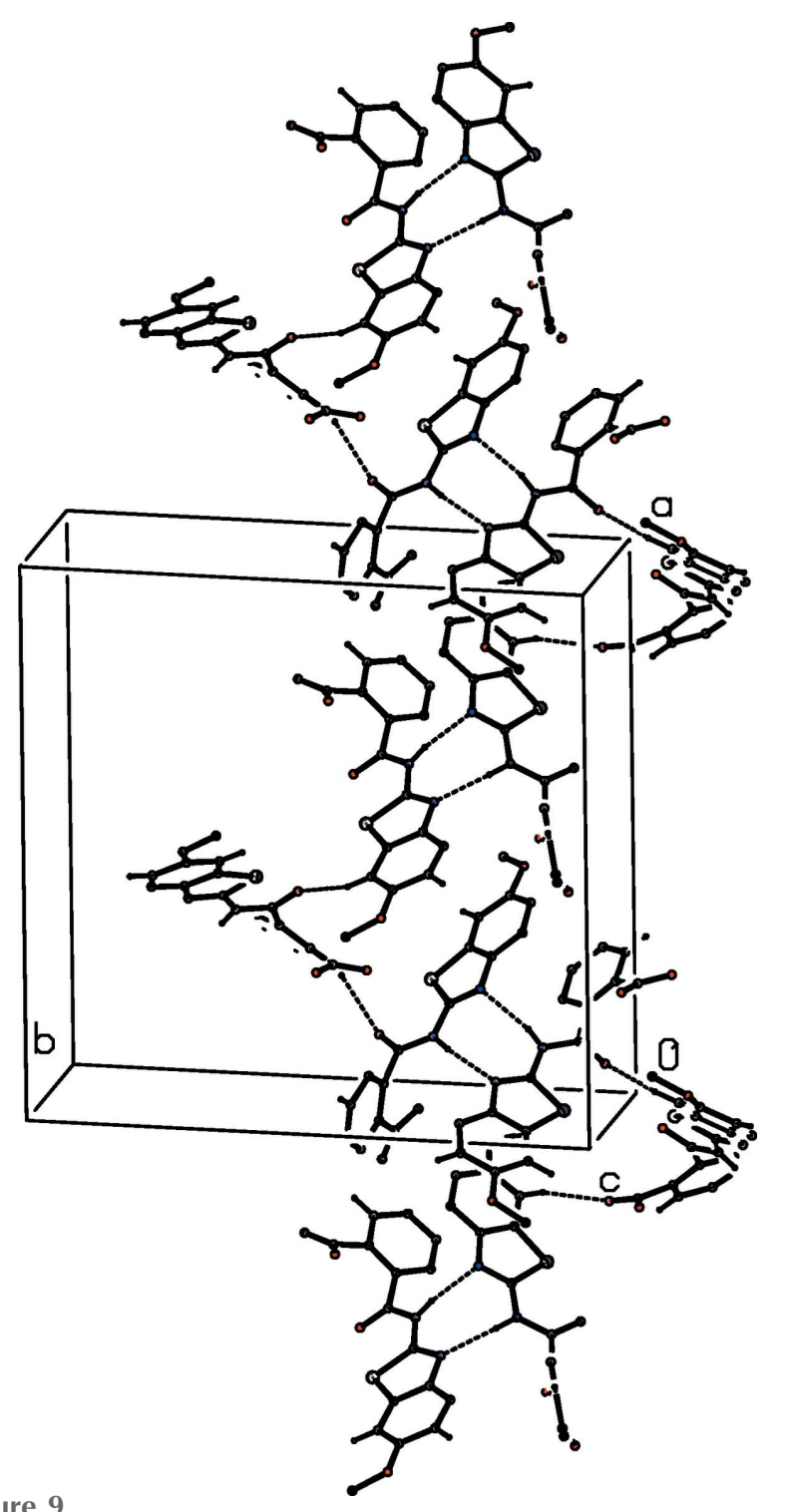

Figure 9

Part of the crystal structure of (II) showing the formation of a chain of rings running parallel to [100] and built from $\mathrm{N}-\mathrm{H} \cdots \mathrm{N}$ and $\mathrm{C}-\mathrm{H} \cdots \mathrm{O}$ hydrogen bonds. Hydrogen bonds are drawn as dashed lines and, for the sake of clarity, the $\mathrm{H}$ atoms not involved in the motifs shown have been omitted. 


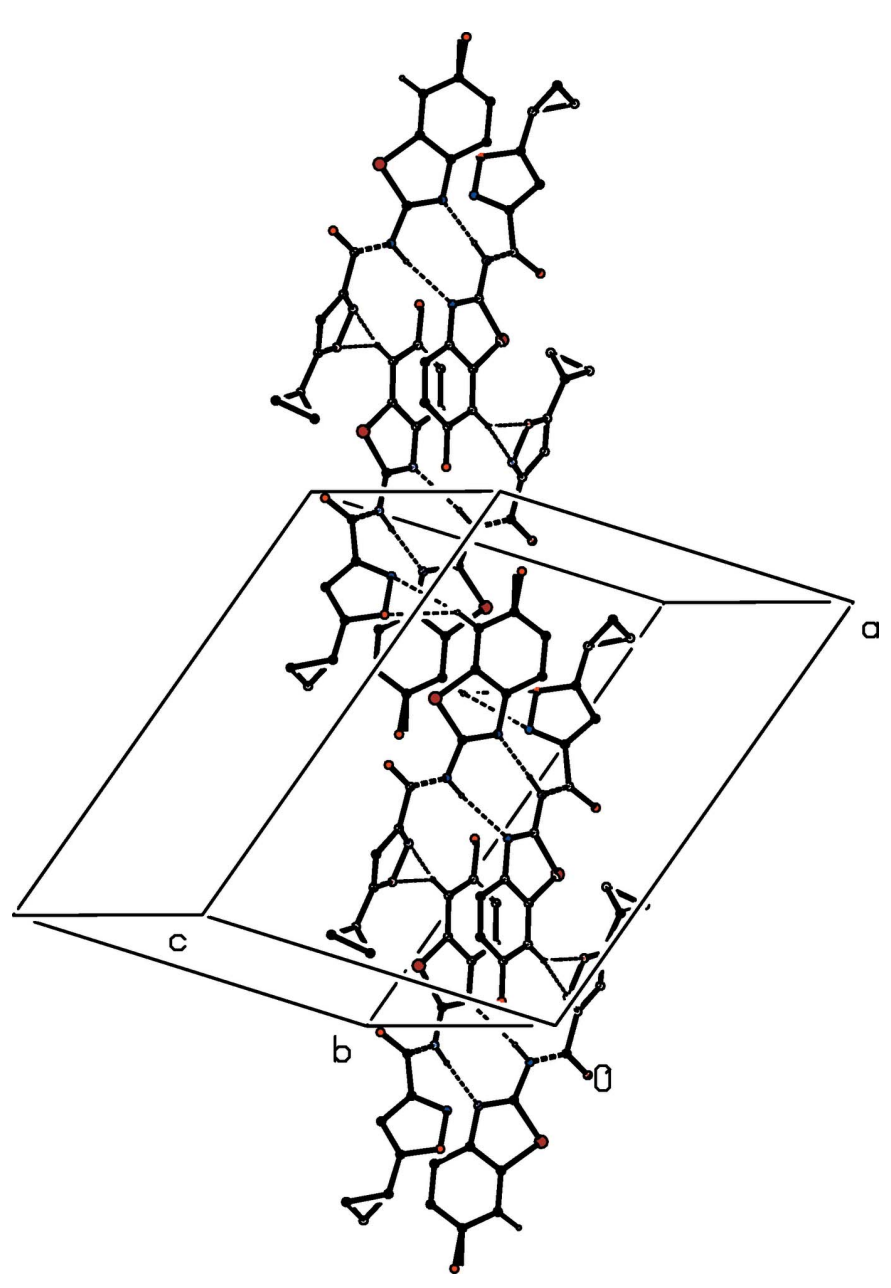

Figure 10

Part of the crystal structure of (III) showing the formation of a chain of rings running parallel to the [101] direction. For the sake of clarity, the methyl groups, the minor disorder component and the $\mathrm{H}$ atoms which are not involved in the motif shown have all been omitted.

linked to the heterocycle at position 6, the structure of (IV) contains no $\mathrm{N}-\mathrm{H} \cdots \mathrm{O}$ hydrogen bonds: instead, inversionrelated pairs of molecules are linked by pairwise $\mathrm{N}-\mathrm{H} \cdots \mathrm{N}$ hydrogen bonds to form cyclic, centrosymmetric $R_{2}^{2}(8)$ dimers. By contrast with (I), there are no short $\mathrm{Br} \cdot \mathrm{Br}$ contacts in the structure of (IV).

In the simple amine 2-amino-6-methylbenzo $[d]$ thiazole, which crystallizes with $Z^{\prime}=2$ in space group $P \overline{1}$ (GINBIP; Saeed et al., 2007), the molecules are linked into complex chains by a combination of three $\mathrm{N}-\mathrm{H} \cdots \mathrm{N}$ hydrogen bonds and one $\mathrm{N}-\mathrm{H} \cdots \pi$ (arene) hydrogen bond, while in the closely related 2-amino-6-nitrobenzo[d]thiazole (TIJLUT; Glidewell et al., 2001), inversion-related molecules are once again linked by pairwise $\mathrm{N}-\mathrm{H} \cdots \mathrm{N}$ hydrogen bonds to form $R_{2}^{2}(8)$ dimers, which are further linked by a three-centre $\mathrm{N}-\mathrm{H} \cdots(\mathrm{O}, \mathrm{O})$ system to form a three-dimensional network.

\section{Synthesis and crystallization}

All reagents were obtained commercially and all were used as received. For the synthesis of compound (I), a solution of triethylamine $(1.11 \mathrm{~g}, 0.01 \mathrm{~mol})$ in dry toluene $(5 \mathrm{ml})$ was added to a mixture of 6-aminobenzo $[d]$ thiazole $(1.50 \mathrm{~g}$, $0.01 \mathrm{~mol})$ and 3-bromobenzoyl chloride $(2.18 \mathrm{~g}, 0.01 \mathrm{~mol})$ in dry toluene $(20 \mathrm{ml})$, and the resulting mixture was heated under reflux for $4 \mathrm{~h}$. When the reaction was complete, as indicated by TLC monitoring, the mixture was cooled to room temperature and the triethylammonium chloride was removed by filtration. The solvent was then removed under reduced pressure and the resulting solid product was washed with water and then crystallized from ethanol solution. Yield $86 \%$, m.p. 439-441 K: IR $\left(\mathrm{cm}^{-1}\right) 3125(\mathrm{~N}-\mathrm{H}), 1667(\mathrm{C}=\mathrm{O}), 1616$ $(\mathrm{C}=\mathrm{N})$; NMR $\left(\mathrm{CDCl}_{3}\right) \delta\left({ }^{1} \mathrm{H}\right) 7.90(s, 1 \mathrm{H}$, thiazole $), 8.21(\mathrm{~s}$, $1 \mathrm{H}), \mathrm{NH}), 6.8-7.9(m, 7 \mathrm{H}$, aromatic); MS (70 eV) $m / z$ 335/333, relative intensities $1: 1\left(M^{+}+1\right)$. Compound (II) was prepared in a similar manner, using 2-amino-6-methoxybenzo[ $d]$ thia]thiazole $(1.80 \mathrm{~g}, 0.01 \mathrm{~mol})$ and 2-nitrobenzoyl chloride (1.85 g, $0.01 \mathrm{~mol})$. Yield 87\%, m.p. 468-470 K; IR $\left(\mathrm{cm}^{-1}\right) 3150$ $(\mathrm{N}-\mathrm{H}), 1681(\mathrm{C}=\mathrm{O}), 1615(\mathrm{C}=\mathrm{N}), 1560$ and 1346 (nitro); NMR $\left(\mathrm{CDCl}_{3}\right) \delta\left({ }^{1} \mathrm{H}\right) 3.80(s, 3 \mathrm{H}, \mathrm{OMe}), 7.2-8.6(m, 7 \mathrm{H}$, aromatic), $8.10(s, 1 \mathrm{H}, \mathrm{NH})$; MS $(70 \mathrm{eV}) \mathrm{m} / z 330\left(M^{+}+1\right)$. Compound (III) was similarly prepared using 2-amino-6methoxybenzo[d]thiazole $(1.80 \mathrm{~g}, 0.01 \mathrm{~mol})$ and 5-cyclopropylisoxazole-3-carboxylchloride $(1.71 \mathrm{~g}, 0.01 \mathrm{~mol})$. Yield 88\%, m.p. 453 K: IR $\left(\mathrm{cm}^{-1}\right) 3120(\mathrm{~N}-\mathrm{H}), 1676(\mathrm{C}=\mathrm{O}), 1625$ $(\mathrm{C}=\mathrm{N})$; NMR (DMSO- $\left.d_{6}\right) \delta\left({ }^{1} \mathrm{H}\right)$ 0.2-2.1 ( $m, 5 \mathrm{H}$, cyclopropyl), $3.83(s, 3 \mathrm{H}, \mathrm{OMe}), 6.90(s, 1 \mathrm{H}, \mathrm{H}-17), 7.20(d, 1 \mathrm{H}, J=7.4 \mathrm{~Hz})$ and $7.46(d, 1 \mathrm{H}, J=7.4 \mathrm{~Hz})((\mathrm{H}-14$ and $\mathrm{H}-15), 7.80(s, 1 \mathrm{H}, \mathrm{H}-$ 4); MS (70 eV) $m / z 316\left(M^{+}+1\right)$.

\section{Refinement}

Crystal data, data collection and structure refinement details are summarized in Table 2 . One bad outlier reflection $(0,23,3)$ was omitted from the final refinement of compound (II). All H atoms, apart from those in the disordered components of compound (III), were located in difference maps. The $\mathrm{H}$ atoms bonded to $\mathrm{C}$ atoms were treated as riding atoms in geometrically idealized positions, with $\mathrm{C}-\mathrm{H}$ distances of $0.93 \AA$ (aromatic and heterocyclic), $0.96 \AA\left(\mathrm{CH}_{3}\right), 0.97 \AA\left(\mathrm{CH}_{2}\right)$ or $0.98 \AA$ (aliphatic $\mathrm{C}-\mathrm{H}$ ) and with $U_{\text {iso }}(\mathrm{H})=k U_{\text {eq }}(\mathrm{C})$, where $k$ $=1.5$ for the methyl groups, which were allowed to rotate but not to tilt, and 1.2 for all other $\mathrm{H}$ atoms bonded to $\mathrm{C}$ atoms. For the $\mathrm{H}$ atoms bonded to $\mathrm{N}$ atoms, the atomic coordinates were refined with $U_{\text {iso }}(\mathrm{H})=1.2 U_{\text {eq }}(\mathrm{N})$, giving the $\mathrm{N}-\mathrm{H}$ distances shown in Table 1. For the disordered methyl group in compound N3, the site occupancies were fixed at 0.5 (see Section 2, above): when these occupancies were refined, the resulting values were $0.504(7)$ and $0.496(7)$, much as expected. For each of the disordered fragments in (III), the corresponding bonded distances and the 1,3 non-bonded distances were restrained to be equal, subject to s.u. values of 0.01 and $0.02 \AA$, respectively. In addition, the anisotropic displacement parameters for corresponding pairs of atoms in the 3-cyclopropyl-5-carbonyloxazole fragments were constrained to be equal. Subject to these conditions, the occupancies of this disordered fragment refined to 0.549 (5) and 0.451 (5). The correct orientation of the structure of the 
Table 2

Experimental details.

(I)

Crystal data

Chemical formula

$M_{\mathrm{r}}$

Crystal system, space group

Temperature (K)

$a, b, c(\AA)$

$\alpha, \beta, \gamma\left({ }^{\circ}\right)$

$V\left(\AA^{3}\right)$

Z

Radiation type

$\mu\left(\mathrm{mm}^{-1}\right)$

Crystal size (mm)

Data collection

Diffractometer

Absorption correction

$T_{\min }, T_{\max }$

No. of measured, independent and observed $[I>2 \sigma(I)]$ reflections

$R_{\text {int }}$

$(\sin \theta / \lambda)_{\max }\left(\AA^{-1}\right)$

$\mathrm{C}_{14} \mathrm{H}_{9} \mathrm{BrN}_{2} \mathrm{OS}$
333.19
Monoclinic, $P 2_{1} / c$
296
$24.221(1), 4.9481(3), 10.9981(6)$
$90,95.371(5), 90$
$1312.31(12)$
4
Mo $K \alpha$
3.28
$0.50 \times 0.36 \times 0.08$

Oxford Diffraction Xcalibur CCD Multi-scan (CrysAlis RED; Oxford Diffraction, 2009)

$0.168,0.769$

$4839,2805,2170$

0.031

0.656

Refinement

$R\left[F^{2}>2 \sigma\left(F^{2}\right)\right], w R\left(F^{2}\right), S$

No. of reflections

No. of parameters

No. of restraints

$\mathrm{H}$-atom treatment

$\Delta \rho_{\max }, \Delta \rho_{\min }\left(\mathrm{e} \AA^{-3}\right)$

Absolute structure

Absolute structure parameter
(II)

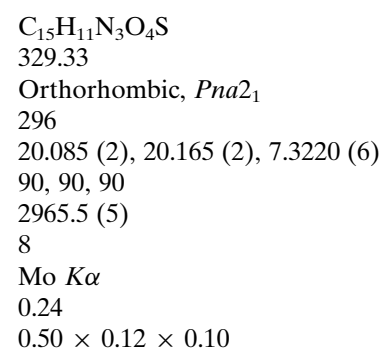

$0.50 \times 0.12 \times 0.10$

Oxford Diffraction Xcalibur CCD

Multi-scan (CrysAlis RED; Oxford

Diffraction, 2009)

$0.787,0.976$

8012, 4784, 2969

0.040

0.661

$0.049,0.075,1.05$
4784
423
1
H atoms treated by a mixture of
$\quad$ independent and constrained
$\quad$ refinement
$0.19,-0.22$
Flack x parameter (Parsons et al.,
$2013)$
$0.02(5)$

(III)

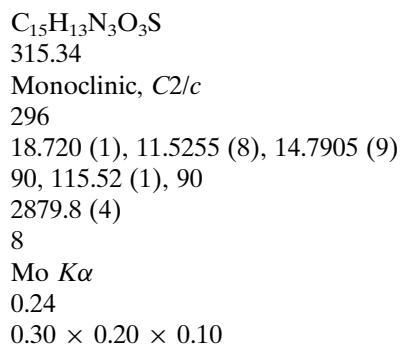

Oxford Diffraction Xcalibur CCD Multi-scan (CrysAlis RED; Oxford Diffraction, 2009) $0.908,0.976$

$5937,3118,1730$

0.038

0.656

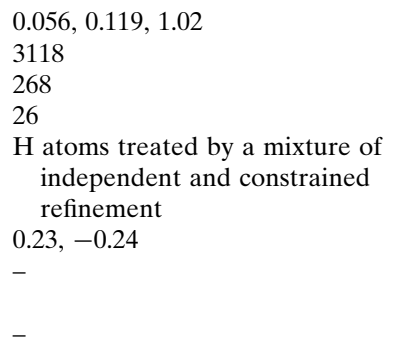

Computer programs: CrysAlis CCD and CrysAlis RED (Oxford Diffraction, 2009), SHELXT (Sheldrick, 2015a), SHELXL2014 (Sheldrick, 2015b) and PLATON (Spek, 2020).

crystal of compound (II) chosen for data collection relative to the polar axis direction was established by means of the Flack $x$ parameter (Flack, 1983); $x=0.02(5)$, calculated (Parsons $e t$ al., 2013) using 708 quotients of the type $\left[\left(I^{+}\right)-\left(I^{-}\right)\right] /$ $\left[\left(I^{+}\right)+\left(I^{-}\right)\right]$.

\section{Acknowledgements}

NM thanks the University of Mysore for research facilities.

\section{Funding information}

HSY is grateful to the UGC, New Delhi, for the award of a BSR Faculty Fellowship for three years.

\section{References}

Bernstein, J., Davis, R. E., Shimoni, L. \& Chang, N.-L. (1995). Angew. Chem. Int. Ed. Engl. 34, 1555-1573.

Cavallo, G., Metrangolo, P., Milan, R., Pilati, T., Priimagi, T., Resnati, G. \& Teraneo, G. (2016). Chem. Rev. 116, 2378-2601.

Etter, M. C. (1990). Acc. Chem. Res. 23, 120-126.

Etter, M. C., MacDonald, J. C. \& Bernstein, J. (1990). Acta Cryst. B46, 256-262.

Ferguson, G., Glidewell, C., Gregson, R. M. \& Meehan, P. R. (1998a). Acta Cryst. B54, 129-138.

Ferguson, G., Glidewell, C., Gregson, R. M. \& Meehan, P. R. (1998b). Acta Cryst. B54, 139-150.
Ferguson, G., Glidewell, C. \& Patterson, I. L. J. (1996). Acta Cryst. C52, 420-423.

Flack, H. D. (1983). Acta Cryst. A39, 876-881.

Fun, H.-K., Quah, C. K., Narayana, B., Nayak, P. S. \& Sarojini, B. K. (2011a). Acta Cryst. E67, o2926-o2927.

Fun, H.-K., Quah, C. K., Narayana, B., Nayak, P. S. \& Sarojini, B. K. (2011b). Acta Cryst. E67, o2941-02942.

Fun, H.-K., Quah, C. K., Nayak, P. S., Narayana, B. \& Sarojini, B. K. (2012). Acta Cryst. E68, o2463.

Fun, H.-K., Shahani, T., Nayak, P. S., Narayana, B. \& Sarojini, B. K. (2012). Acta Cryst. E68, o519.

Gao, K., Liu, J., Zuo, K., Feng, X. \& Gao, Y. (2020). Molecules, 25, 1675-1690.

Glidewell, C., Low, J. N., McWilliam, S. A., Skakle, J. M. S. \& Wardell, J. L. (2001). Acta Cryst. C57, 1209-1211.

Gregson, R. M., Glidewell, C., Ferguson, G. \& Lough, A. J. (2000). Acta Cryst. B56, 39-57.

Groom, C. R., Bruno, I. J., Lightfoot, M. P. \& Ward, S. C. (2016). Acta Cryst. B72, 171-179.

Henary, M., Paranjpe, S. \& Owens, E. A. (2013). Heterocycl. Commun. 19, 89-99.

Imramovský, A., Pejchal, V., Štěpánková, Š., Vorčáková, K., Jampílek, J., Vančo, J., Šimůnek, P., Královec, K., Brůčková, L., Mandíková, J. \& Trejtnar, F. (2013). Bioorg. Med. Chem. 21, 1735-1748.

Jung, K.-Y., Kim, S.-K., Gao, Z.-G., Gross, A. S., Melman, N., Jacobson, K. A. \& Kim, Y.-C. (2004). Bioorg. Med. Chem. 12, 613623.

Nayak, P. S., Jasinski, J. P., Golen, J. A., Narayana, B., Kaur, M., Yathirajan, H. S. \& Glidewell, C. (2014). Acta Cryst. C70, 889-894. 
Odame, F., Woodcock, G., Hosten, E. C., Lobb, K. \& Tshentu, Z. R. (2020). J. Organomet. Chem. 922, 121359.

Oxford Diffraction (2009). CrysAlis CCD and CrysAlis RED. Oxford Diffraction Ltd, Abingdon, England.

Parsons, S., Flack, H. D. \& Wagner, T. (2013). Acta Cryst. B69, 249259.

Praveen, A. S., Jasinski, J. P., Golen, J. A., Narayana, B. \& Yathirajan, H. S. (2011). Acta Cryst. E67, o1826.

Praveen, A. S., Yathirajan, H. S., Jasinski, J. P., Keeley, A. C., Narayana, B. \& Sarojini, B. K. (2013a). Acta Cryst. E69, o900-o901.

Praveen, A. S., Yathirajan, H. S., Jasinski, J. P., Keeley, A. C., Narayana, B. \& Sarojini, B. K. (2013b). Acta Cryst. E69, o996-o997.
Ramasubbu, N., Parthasarathy, R. \& Murray-Rust, P. (1986). J. Am. Chem. Soc. 108, 4308-4314.

Rowland, R. S. \& Taylor, R. (1996). J. Phys. Chem. 100, 73847391.

Saeed, A., Rafique, H. \& Bolte, M. (2007). Acta Cryst. E63, o4247. Seip, H. M. \& Seip, R. (1973). Acta Chem. Scand. 27, 4024-4027.

Sheldrick, G. M. (2015a). Acta Cryst. A71, 3-8.

Sheldrick, G. M. (2015b). Acta Cryst. C71, 3-8.

Spek, A. L. (2020). Acta Cryst. E76, 1-11.

White, E. H., McCapra, F. \& Field, G. F. (1963). J. Am. Chem. Soc. 85, 337-343. 


\section{supporting information}

Acta Cryst. (2021). E77, 504-511 [https://doi.org/10.1107/S2056989021003637]

Different patterns of supramolecular aggregation in three amides containing $N$-(benzo[d] thiazolyl) substituents

Ninganayaka Mahesha, Hemmige S. Yathirajan, Holalagudu A. Nagma Banu, Balakrishna

Kalluraya, Sabine Foro and Christopher Glidewell

Computing details

For all structures, data collection: CrysAlis CCD (Oxford Diffraction, 2009); cell refinement: CrysAlis RED (Oxford Diffraction, 2009); data reduction: CrysAlis RED (Oxford Diffraction, 2009); program(s) used to solve structure:

SHELXT (Sheldrick, 2015a); program(s) used to refine structure: SHELXL2014 (Sheldrick, 2015b); molecular graphics: PLATON (Spek, 2020); software used to prepare material for publication: SHELXL2014 (Sheldrick, 2015b) and PLATON (Spek, 2020).

N-(Benzo[d]thiazol-6-yl)-3-bromobenzamide (I)

Crystal data

$\mathrm{C}_{14} \mathrm{H}_{9} \mathrm{BrN}_{2} \mathrm{OS}$

$M_{r}=333.19$

Monoclinic, $P 2_{1} / c$

$a=24.221(1) \AA$

$b=4.9481(3) \AA$

$c=10.9981(6) \AA$

$\beta=95.371(5)^{\circ}$

$V=1312.31(12) \AA^{3}$

$Z=4$

Data collection

Oxford Diffraction Xcalibur CCD diffractometer

Radiation source: Enhance (Mo) X-ray Source

Graphite monochromator

$\omega$ scans

Absorption correction: multi-scan

(CrysalisRed; Oxford Diffraction, 2009)

$T_{\min }=0.168, T_{\max }=0.769$

Refinement

Refinement on $F^{2}$

Least-squares matrix: full

$R\left[F^{2}>2 \sigma\left(F^{2}\right)\right]=0.043$

$w R\left(F^{2}\right)=0.123$

$S=1.03$

2805 reflections

175 parameters
$F(000)=664$

$D_{\mathrm{x}}=1.686 \mathrm{Mg} \mathrm{m}^{-3}$

Mo $K \alpha$ radiation, $\lambda=0.71073 \AA$

Cell parameters from 2805 reflections

$\theta=2.5-27.8^{\circ}$

$\mu=3.28 \mathrm{~mm}^{-1}$

$T=296 \mathrm{~K}$

Plate, colourless

$0.50 \times 0.36 \times 0.08 \mathrm{~mm}$

4839 measured reflections

2805 independent reflections

2170 reflections with $I>2 \sigma(I)$

$R_{\text {int }}=0.031$

$\theta_{\max }=27.8^{\circ}, \theta_{\min }=2.5^{\circ}$

$h=-31 \rightarrow 24$

$k=-4 \rightarrow 6$

$l=-10 \rightarrow 14$

0 restraints

Primary atom site location: difference Fourier map

Hydrogen site location: mixed

$\mathrm{H}$ atoms treated by a mixture of independent and constrained refinement 
$w=1 /\left[\sigma^{2}\left(F_{\mathrm{o}}^{2}\right)+(0.0826 P)^{2}\right]$

where $P=\left(F_{\mathrm{o}}^{2}+2 F_{\mathrm{c}}^{2}\right) / 3$

$(\Delta / \sigma)_{\max }=0.001$

$$
\Delta \rho_{\max }=1.00 \mathrm{e} \AA^{-3}
$$

$\Delta \rho_{\min }=-0.73$ e $\AA^{-3}$

Special details

Geometry. All esds (except the esd in the dihedral angle between two 1.s. planes) are estimated using the full covariance matrix. The cell esds are taken into account individually in the estimation of esds in distances, angles and torsion angles; correlations between esds in cell parameters are only used when they are defined by crystal symmetry. An approximate (isotropic) treatment of cell esds is used for estimating esds involving 1.s. planes.

Fractional atomic coordinates and isotropic or equivalent isotropic displacement parameters $\left(\AA^{2}\right)$

\begin{tabular}{lllll}
\hline & $x$ & $y$ & $z$ & $U_{\text {iso }} * / U_{\text {eq }}$ \\
\hline C1 & $0.82689(14)$ & $0.5633(6)$ & $0.8202(3)$ & $0.0285(7)$ \\
C2 & $0.86414(13)$ & $0.3673(6)$ & $0.7894(3)$ & $0.0292(7)$ \\
H2 & 0.8588 & 0.2773 & 0.7150 & $0.035^{*}$ \\
C3 & $0.90946(13)$ & $0.3081(7)$ & $0.8714(3)$ & $0.0300(7)$ \\
Br3 & $0.96192(2)$ & $0.05035(8)$ & $0.82512(3)$ & $0.04011(16)$ \\
C4 & $0.91689(15)$ & $0.4331(7)$ & $0.9832(3)$ & $0.0387(8)$ \\
H4 & 0.9468 & 0.3866 & 1.0383 & $0.046^{*}$ \\
C5 & $0.88018(16)$ & $0.6257(8)$ & $1.0131(3)$ & $0.0422(9)$ \\
H5 & 0.8855 & 0.7119 & 1.0884 & $0.051^{*}$ \\
C6 & $0.83486(14)$ & $0.6942(7)$ & $0.9320(3)$ & $0.0375(8)$ \\
H6 & 0.8101 & 0.8266 & 0.9525 & $0.045^{*}$ \\
C11 & $0.77814(13)$ & $0.6452(7)$ & $0.7322(3)$ & $0.0307(7)$ \\
O11 & $0.76470(12)$ & $0.8823(5)$ & $0.7208(3)$ & $0.0490(7)$ \\
N11 & $0.75247(12)$ & $0.4425(6)$ & $0.6688(3)$ & $0.0327(6)$ \\
H11 & $0.7634(15)$ & $0.274(7)$ & $0.689(3)$ & $0.039^{*}$ \\
S11 & $0.55928(5)$ & $0.8448(3)$ & $0.49883(12)$ & $0.0659(4)$ \\
C12 & $0.53824(18)$ & $0.6976(10)$ & $0.3595(4)$ & $0.0604(12)$ \\
H12 & 0.5048 & 0.7447 & 0.3163 & $0.072^{*}$ \\
N13 & $0.57051(15)$ & $0.5206(8)$ & $0.3178(3)$ & $0.0594(10)$ \\
C13A & $0.61798(16)$ & $0.4886(8)$ & $0.4010(3)$ & $0.0421(9)$ \\
C14 & $0.66075(17)$ & $0.3153(10)$ & $0.3870(3)$ & $0.0530(11)$ \\
H14 & 0.6603 & 0.2073 & 0.3177 & $0.064^{*}$ \\
C15 & $0.70478(16)$ & $0.3012(8)$ & $0.4767(3)$ & $0.0455(9)$ \\
H15 & 0.7336 & 0.1800 & 0.4687 & $0.055^{*}$ \\
C16 & $0.70600(14)$ & $0.4690(7)$ & $0.5795(3)$ & $0.0310(7)$ \\
C17 & $0.66363(14)$ & $0.6445(7)$ & $0.5954(3)$ & $0.0375(8)$ \\
H17 & 0.6645 & 0.7552 & 0.6639 & $0.045^{*}$ \\
C17A & $0.61882(14)$ & $0.6514(8)$ & $0.5046(3)$ & $0.0397(8)$ \\
& & & &
\end{tabular}

Atomic displacement parameters $\left(A^{2}\right)$

\begin{tabular}{lllllll}
\hline & $U^{11}$ & $U^{22}$ & $U^{33}$ & $U^{12}$ & $U^{13}$ & $U^{23}$ \\
\hline C1 & $0.0266(16)$ & $0.0218(16)$ & $0.0363(16)$ & $0.0008(13)$ & $-0.0016(12)$ & $0.0046(14)$ \\
C2 & $0.0307(17)$ & $0.0245(16)$ & $0.0313(16)$ & $-0.0011(13)$ & $-0.0026(12)$ & $0.0009(13)$ \\
C3 & $0.0270(16)$ & $0.0284(17)$ & $0.0338(16)$ & $0.0008(14)$ & $-0.0008(12)$ & $0.0076(14)$ \\
Br3 & $0.0313(2)$ & $0.0432(3)$ & $0.0450(2)$ & $0.01066(16)$ & $-0.00006(14)$ & $0.00559(16)$
\end{tabular}




\begin{tabular}{lllllll} 
C4 & $0.040(2)$ & $0.045(2)$ & $0.0296(16)$ & $-0.0045(17)$ & $-0.0045(14)$ & $0.0042(16)$ \\
C5 & $0.047(2)$ & $0.046(2)$ & $0.0321(18)$ & $0.0028(18)$ & $-0.0032(15)$ & $-0.0062(16)$ \\
C6 & $0.038(2)$ & $0.035(2)$ & $0.0389(18)$ & $0.0046(16)$ & $0.0054(14)$ & $-0.0022(16)$ \\
C11 & $0.0263(17)$ & $0.0246(16)$ & $0.0404(18)$ & $0.0033(14)$ & $-0.0011(13)$ & $0.0048(14)$ \\
O11 & $0.0486(17)$ & $0.0207(12)$ & $0.0733(19)$ & $0.0058(11)$ & $-0.0175(13)$ & $0.0014(12)$ \\
N11 & $0.0316(16)$ & $0.0199(14)$ & $0.0444(16)$ & $0.0061(12)$ & $-0.0078(12)$ & $0.0036(13)$ \\
S11 & $0.0404(6)$ & $0.0748(8)$ & $0.0777(8)$ & $0.0279(6)$ & $-0.0188(5)$ & $-0.0133(7)$ \\
C12 & $0.038(2)$ & $0.077(3)$ & $0.062(3)$ & $0.013(2)$ & $-0.0154(18)$ & $0.006(2)$ \\
N13 & $0.039(2)$ & $0.087(3)$ & $0.049(2)$ & $0.0027(19)$ & $-0.0172(16)$ & $0.0004(19)$ \\
C13A & $0.036(2)$ & $0.054(2)$ & $0.0348(18)$ & $0.0020(17)$ & $-0.0072(15)$ & $0.0039(16)$ \\
C14 & $0.045(2)$ & $0.069(3)$ & $0.043(2)$ & $0.008(2)$ & $-0.0041(16)$ & $-0.016(2)$ \\
C15 & $0.036(2)$ & $0.047(2)$ & $0.052(2)$ & $0.0107(18)$ & $-0.0015(16)$ & $-0.0086(19)$ \\
C16 & $0.0284(17)$ & $0.0274(17)$ & $0.0358(17)$ & $0.0020(14)$ & $-0.0047(13)$ & $0.0043(14)$ \\
C17 & $0.0339(19)$ & $0.0341(18)$ & $0.0426(19)$ & $0.0060(16)$ & $-0.0063(14)$ & $-0.0040(16)$ \\
C17A & $0.0290(18)$ & $0.041(2)$ & $0.047(2)$ & $0.0100(16)$ & $-0.0061(14)$ & $0.0043(17)$ \\
& & & & & & \\
\hline
\end{tabular}

Geometric parameters $\left(A,{ }^{o}\right)$

\begin{tabular}{|c|c|c|c|}
\hline $\mathrm{C} 1-\mathrm{C} 6$ & $1.387(4)$ & N11-H11 & $0.90(4)$ \\
\hline $\mathrm{C} 1-\mathrm{C} 2$ & $1.388(5)$ & $\mathrm{S} 11-\mathrm{C} 17 \mathrm{~A}$ & $1.727(4)$ \\
\hline $\mathrm{C} 1-\mathrm{C} 11$ & $1.510(4)$ & $\mathrm{S} 11-\mathrm{C} 12$ & $1.730(5)$ \\
\hline $\mathrm{C} 2-\mathrm{C} 3$ & $1.385(4)$ & $\mathrm{C} 12-\mathrm{N} 13$ & $1.287(6)$ \\
\hline $\mathrm{C} 2-\mathrm{H} 2$ & 0.9300 & $\mathrm{C} 12-\mathrm{H} 12$ & 0.9300 \\
\hline $\mathrm{C} 3-\mathrm{C} 4$ & $1.373(5)$ & $\mathrm{N} 13-\mathrm{C} 13 \mathrm{~A}$ & $1.410(5)$ \\
\hline $\mathrm{C} 3-\mathrm{Br} 3$ & $1.903(3)$ & $\mathrm{C} 13 \mathrm{~A}-\mathrm{C} 14$ & $1.365(6)$ \\
\hline $\mathrm{C} 4-\mathrm{C} 5$ & $1.364(5)$ & $\mathrm{C} 13 \mathrm{~A}-\mathrm{C} 17 \mathrm{~A}$ & $1.394(5)$ \\
\hline $\mathrm{C} 4-\mathrm{H} 4$ & 0.9300 & $\mathrm{C} 14-\mathrm{C} 15$ & $1.385(5)$ \\
\hline $\mathrm{C} 5-\mathrm{C} 6$ & $1.390(5)$ & $\mathrm{C} 14-\mathrm{H} 14$ & 0.9300 \\
\hline $\mathrm{C} 5-\mathrm{H} 5$ & 0.9300 & $\mathrm{C} 15-\mathrm{C} 16$ & $1.400(5)$ \\
\hline C6- 6 6 & 0.9300 & $\mathrm{C} 15-\mathrm{H} 15$ & 0.9300 \\
\hline $\mathrm{C} 11-\mathrm{O} 11$ & $1.221(4)$ & $\mathrm{C} 16-\mathrm{C} 17$ & $1.368(5)$ \\
\hline $\mathrm{C} 11-\mathrm{N} 11$ & $1.341(4)$ & $\mathrm{C} 17-\mathrm{C} 17 \mathrm{~A}$ & $1.405(4)$ \\
\hline $\mathrm{N} 11-\mathrm{C} 16$ & $1.429(4)$ & $\mathrm{C} 17-\mathrm{H} 17$ & 0.9300 \\
\hline $\mathrm{C} 6-\mathrm{C} 1-\mathrm{C} 2$ & $120.2(3)$ & $\mathrm{C} 17 \mathrm{~A}-\mathrm{S} 11-\mathrm{C} 12$ & $88.6(2)$ \\
\hline $\mathrm{C} 6-\mathrm{C} 1-\mathrm{C} 11$ & $118.6(3)$ & $\mathrm{N} 13-\mathrm{C} 12-\mathrm{S} 11$ & $117.6(3)$ \\
\hline $\mathrm{C} 2-\mathrm{C} 1-\mathrm{C} 11$ & $121.2(3)$ & $\mathrm{N} 13-\mathrm{C} 12-\mathrm{H} 12$ & 121.2 \\
\hline $\mathrm{C} 3-\mathrm{C} 2-\mathrm{C} 1$ & $118.8(3)$ & $\mathrm{S} 11-\mathrm{C} 12-\mathrm{H} 12$ & 121.2 \\
\hline $\mathrm{C} 3-\mathrm{C} 2-\mathrm{H} 2$ & 120.6 & $\mathrm{C} 12-\mathrm{N} 13-\mathrm{C} 13 \mathrm{~A}$ & $109.3(4)$ \\
\hline $\mathrm{C} 1-\mathrm{C} 2-\mathrm{H} 2$ & 120.6 & $\mathrm{C} 14-\mathrm{C} 13 \mathrm{~A}-\mathrm{C} 17 \mathrm{~A}$ & $120.1(3)$ \\
\hline $\mathrm{C} 4-\mathrm{C} 3-\mathrm{C} 2$ & $121.2(3)$ & $\mathrm{C} 14-\mathrm{C} 13 \mathrm{~A}-\mathrm{N} 13$ & $125.4(4)$ \\
\hline $\mathrm{C} 4-\mathrm{C} 3-\mathrm{Br} 3$ & $120.4(2)$ & $\mathrm{C} 17 \mathrm{~A}-\mathrm{C} 13 \mathrm{~A}-\mathrm{N} 13$ & $114.5(4)$ \\
\hline $\mathrm{C} 2-\mathrm{C} 3-\mathrm{Br} 3$ & $118.4(2)$ & $\mathrm{C} 13 \mathrm{~A}-\mathrm{C} 14-\mathrm{C} 15$ & $119.6(4)$ \\
\hline $\mathrm{C} 5-\mathrm{C} 4-\mathrm{C} 3$ & $119.8(3)$ & $\mathrm{C} 13 \mathrm{~A}-\mathrm{C} 14-\mathrm{H} 14$ & 120.2 \\
\hline $\mathrm{C} 5-\mathrm{C} 4-\mathrm{H} 4$ & 120.1 & $\mathrm{C} 15-\mathrm{C} 14-\mathrm{H} 14$ & 120.2 \\
\hline $\mathrm{C} 3-\mathrm{C} 4-\mathrm{H} 4$ & 120.1 & $\mathrm{C} 14-\mathrm{C} 15-\mathrm{C} 16$ & $120.1(3)$ \\
\hline $\mathrm{C} 4-\mathrm{C} 5-\mathrm{C} 6$ & $120.6(3)$ & $\mathrm{C} 14-\mathrm{C} 15-\mathrm{H} 15$ & 120.0 \\
\hline $\mathrm{C} 4-\mathrm{C} 5-\mathrm{H} 5$ & 119.7 & $\mathrm{C} 16-\mathrm{C} 15-\mathrm{H} 15$ & 120.0 \\
\hline
\end{tabular}




\begin{tabular}{|c|c|c|c|}
\hline $\mathrm{C} 6-\mathrm{C} 5-\mathrm{H} 5$ & 119.7 & $\mathrm{C} 17-\mathrm{C} 16-\mathrm{C} 15$ & $121.4(3)$ \\
\hline $\mathrm{C} 1-\mathrm{C} 6-\mathrm{C} 5$ & $119.4(3)$ & $\mathrm{C} 17-\mathrm{C} 16-\mathrm{N} 11$ & $121.5(3)$ \\
\hline $\mathrm{C} 1-\mathrm{C} 6-\mathrm{H} 6$ & 120.3 & $\mathrm{C} 15-\mathrm{C} 16-\mathrm{N} 11$ & $117.1(3)$ \\
\hline $\mathrm{C} 5-\mathrm{C} 6-\mathrm{H} 6$ & 120.3 & $\mathrm{C} 16-\mathrm{C} 17-\mathrm{C} 17 \mathrm{~A}$ & $117.6(3)$ \\
\hline $\mathrm{O} 11-\mathrm{C} 11-\mathrm{N} 11$ & $124.0(3)$ & $\mathrm{C} 16-\mathrm{C} 17-\mathrm{H} 17$ & 121.2 \\
\hline $\mathrm{O} 11-\mathrm{C} 11-\mathrm{C} 1$ & $120.6(3)$ & $\mathrm{C} 17 \mathrm{~A}-\mathrm{C} 17-\mathrm{H} 17$ & 121.2 \\
\hline $\mathrm{N} 11-\mathrm{C} 11-\mathrm{C} 1$ & $115.4(3)$ & $\mathrm{C} 13 \mathrm{~A}-\mathrm{C} 17 \mathrm{~A}-\mathrm{C} 17$ & $121.2(3)$ \\
\hline $\mathrm{C} 11-\mathrm{N} 11-\mathrm{C} 16$ & $125.9(3)$ & $\mathrm{C} 13 \mathrm{~A}-\mathrm{C} 17 \mathrm{~A}-\mathrm{S} 11$ & $110.0(3)$ \\
\hline $\mathrm{C} 11-\mathrm{N} 11-\mathrm{H} 11$ & $117(2)$ & $\mathrm{C} 17-\mathrm{C} 17 \mathrm{~A}-\mathrm{S} 11$ & $128.8(3)$ \\
\hline $\mathrm{C} 16-\mathrm{N} 11-\mathrm{H} 11$ & $117(2)$ & & \\
\hline $\mathrm{C} 6-\mathrm{C} 1-\mathrm{C} 2-\mathrm{C} 3$ & $-0.8(5)$ & $\mathrm{C} 12-\mathrm{N} 13-\mathrm{C} 13 \mathrm{~A}-\mathrm{C} 17 \mathrm{~A}$ & $-0.3(6)$ \\
\hline $\mathrm{C} 11-\mathrm{C} 1-\mathrm{C} 2-\mathrm{C} 3$ & $177.2(3)$ & $\mathrm{C} 17 \mathrm{~A}-\mathrm{C} 13 \mathrm{~A}-\mathrm{C} 14-\mathrm{C} 15$ & $0.4(7)$ \\
\hline $\mathrm{C} 1-\mathrm{C} 2-\mathrm{C} 3-\mathrm{C} 4$ & $2.2(5)$ & $\mathrm{N} 13-\mathrm{C} 13 \mathrm{~A}-\mathrm{C} 14-\mathrm{C} 15$ & $-179.2(4)$ \\
\hline $\mathrm{C} 1-\mathrm{C} 2-\mathrm{C} 3-\mathrm{Br} 3$ & $-177.3(2)$ & $\mathrm{C} 13 \mathrm{~A}-\mathrm{C} 14-\mathrm{C} 15-\mathrm{C} 16$ & $-1.7(7)$ \\
\hline $\mathrm{C} 2-\mathrm{C} 3-\mathrm{C} 4-\mathrm{C} 5$ & $-2.2(5)$ & $\mathrm{C} 14-\mathrm{C} 15-\mathrm{C} 16-\mathrm{C} 17$ & $1.6(6)$ \\
\hline $\mathrm{Br} 3-\mathrm{C} 3-\mathrm{C} 4-\mathrm{C} 5$ & $177.3(3)$ & $\mathrm{C} 14-\mathrm{C} 15-\mathrm{C} 16-\mathrm{N} 11$ & $179.6(4)$ \\
\hline $\mathrm{C} 3-\mathrm{C} 4-\mathrm{C} 5-\mathrm{C} 6$ & $0.8(6)$ & $\mathrm{C} 11-\mathrm{N} 11-\mathrm{C} 16-\mathrm{C} 17$ & $-39.9(5)$ \\
\hline $\mathrm{C} 2-\mathrm{C} 1-\mathrm{C} 6-\mathrm{C} 5$ & $-0.5(5)$ & $\mathrm{C} 11-\mathrm{N} 11-\mathrm{C} 16-\mathrm{C} 15$ & $142.2(4)$ \\
\hline $\mathrm{C} 11-\mathrm{C} 1-\mathrm{C} 6-\mathrm{C} 5$ & $-178.6(3)$ & $\mathrm{C} 15-\mathrm{C} 16-\mathrm{C} 17-\mathrm{C} 17 \mathrm{~A}$ & $-0.3(6)$ \\
\hline $\mathrm{C} 4-\mathrm{C} 5-\mathrm{C} 6-\mathrm{C} 1$ & $0.5(6)$ & $\mathrm{N} 11-\mathrm{C} 16-\mathrm{C} 17-\mathrm{C} 17 \mathrm{~A}$ & $-178.1(3)$ \\
\hline $\mathrm{C} 6-\mathrm{C} 1-\mathrm{C} 11-\mathrm{O} 11$ & $39.0(5)$ & $\mathrm{C} 14-\mathrm{C} 13 \mathrm{~A}-\mathrm{C} 17 \mathrm{~A}-\mathrm{C} 17$ & $1.0(6)$ \\
\hline $\mathrm{C} 2-\mathrm{C} 1-\mathrm{C} 11-\mathrm{O} 11$ & $-139.0(4)$ & $\mathrm{N} 13-\mathrm{C} 13 \mathrm{~A}-\mathrm{C} 17 \mathrm{~A}-\mathrm{C} 17$ & $-179.4(4)$ \\
\hline $\mathrm{C} 6-\mathrm{C} 1-\mathrm{C} 11-\mathrm{N} 11$ & $-141.9(3)$ & $\mathrm{C} 14-\mathrm{C} 13 \mathrm{~A}-\mathrm{C} 17 \mathrm{~A}-\mathrm{S} 11$ & $-179.1(3)$ \\
\hline $\mathrm{C} 2-\mathrm{C} 1-\mathrm{C} 11-\mathrm{N} 11$ & $40.0(4)$ & $\mathrm{N} 13-\mathrm{C} 13 \mathrm{~A}-\mathrm{C} 17 \mathrm{~A}-\mathrm{S} 11$ & $0.6(5)$ \\
\hline $\mathrm{O} 11-\mathrm{C} 11-\mathrm{N} 11-\mathrm{C} 16$ & $-0.2(6)$ & $\mathrm{C} 16-\mathrm{C} 17-\mathrm{C} 17 \mathrm{~A}-\mathrm{C} 13 \mathrm{~A}$ & $-1.0(6)$ \\
\hline $\mathrm{C} 1-\mathrm{C} 11-\mathrm{N} 11-\mathrm{C} 16$ & $-179.2(3)$ & $\mathrm{C} 16-\mathrm{C} 17-\mathrm{C} 17 \mathrm{~A}-\mathrm{S} 11$ & $179.0(3)$ \\
\hline $\mathrm{C} 17 \mathrm{~A}-\mathrm{S} 11-\mathrm{C} 12-\mathrm{N} 13$ & $0.3(4)$ & $\mathrm{C} 12-\mathrm{S} 11-\mathrm{C} 17 \mathrm{~A}-\mathrm{C} 13 \mathrm{~A}$ & $-0.5(3)$ \\
\hline $\mathrm{S} 11-\mathrm{C} 12-\mathrm{N} 13-\mathrm{C} 13 \mathrm{~A}$ & $0.0(6)$ & $\mathrm{C} 12-\mathrm{S} 11-\mathrm{C} 17 \mathrm{~A}-\mathrm{C} 17$ & $179.5(4)$ \\
\hline $\mathrm{C} 12-\mathrm{N} 13-\mathrm{C} 13 \mathrm{~A}-\mathrm{C} 14$ & $179.3(4)$ & & \\
\hline
\end{tabular}

Hydrogen-bond geometry $\left(\AA,{ }^{\circ}\right)$

\begin{tabular}{lllll}
\hline$D-\mathrm{H} \cdots A$ & $D-\mathrm{H}$ & $\mathrm{H} \cdots A$ & $D \cdots A$ & $D-\mathrm{H} \cdots A$ \\
\hline $\mathrm{N} 11-\mathrm{H} 11 \cdots \mathrm{O} 11^{\mathrm{i}}$ & $0.90(4)$ & $1.97(3)$ & $2.840(4)$ & $164(3)$ \\
$\mathrm{C} 12-\mathrm{H} 12 \cdots \mathrm{N} 13^{\mathrm{ii}}$ & 0.93 & 2.62 & $3.512(6)$ & 161 \\
\hline
\end{tabular}

Symmetry codes: (i) $x, y-1, z$; (ii) $-x+1, y+1 / 2,-z+1 / 2$.

$N$-(6-Methoxybenzo[d] thiazol-2-yl)-2-nitrobenzamide (II)

\section{Crystal data}

$\mathrm{C}_{15} \mathrm{H}_{11} \mathrm{~N}_{3} \mathrm{O}_{4} \mathrm{~S}$

$M_{r}=329.33$

Orthorhombic, Pna2 ${ }_{1}$

$a=20.085$ (2) $\AA$

$b=20.165$ (2) $\AA$

$c=7.3220(6) \AA$

$V=2965.5(5) \AA^{3}$

$Z=8$

$F(000)=1360$
$D_{\mathrm{x}}=1.475 \mathrm{Mg} \mathrm{m}^{-3}$

Mo $K \alpha$ radiation, $\lambda=0.71073 \AA$

Cell parameters from 4785 reflections

$\theta=2.9-28.8^{\circ}$

$\mu=0.24 \mathrm{~mm}^{-1}$

$T=296 \mathrm{~K}$

Needle, yellow

$0.50 \times 0.12 \times 0.10 \mathrm{~mm}$ 


\section{Data collection}

Oxford Diffraction Xcalibur CCD diffractometer

Radiation source: Enhance (Mo) X-ray Source Graphite monochromator $\omega$ scans

Absorption correction: multi-scan (CrysalisRed; Oxford Diffraction, 2009)

$T_{\min }=0.787, T_{\max }=0.976$

\section{Refinement}

Refinement on $F^{2}$

Least-squares matrix: full

$R\left[F^{2}>2 \sigma\left(F^{2}\right)\right]=0.049$

$w R\left(F^{2}\right)=0.075$

$S=1.05$

4784 reflections

423 parameters

1 restraint

Primary atom site location: difference Fourier map

Hydrogen site location: mixed
8012 measured reflections

4784 independent reflections

2969 reflections with $I>2 \sigma(I)$

$R_{\text {int }}=0.040$

$\theta_{\text {max }}=28.0^{\circ}, \theta_{\text {min }}=2.9^{\circ}$

$h=-22 \rightarrow 25$

$k=-25 \rightarrow 23$

$l=-3 \rightarrow 9$

$\mathrm{H}$ atoms treated by a mixture of independent and constrained refinement

$w=1 /\left[\sigma^{2}\left(F_{\mathrm{o}}^{2}\right)+(0.019 P)^{2}+0.5603 P\right]$

where $P=\left(F_{\mathrm{o}}^{2}+2 F_{\mathrm{c}}^{2}\right) / 3$

$(\Delta / \sigma)_{\max }<0.001$

$\Delta \rho_{\max }=0.19 \mathrm{e} \AA^{-3}$

$\Delta \rho_{\min }=-0.22$ e $\AA^{-3}$

Absolute structure: Flack x parameter (Parsons et al., 2013)

Absolute structure parameter: 0.02 (5)

Special details

Geometry. All esds (except the esd in the dihedral angle between two 1.s. planes) are estimated using the full covariance matrix. The cell esds are taken into account individually in the estimation of esds in distances, angles and torsion angles; correlations between esds in cell parameters are only used when they are defined by crystal symmetry. An approximate (isotropic) treatment of cell esds is used for estimating esds involving 1.s. planes.

Fractional atomic coordinates and isotropic or equivalent isotropic displacement parameters $\left(\AA^{2}\right)$

\begin{tabular}{lllll}
\hline & $x$ & $y$ & $z$ & $U_{\text {iso }} * / U_{\text {eq }}$ \\
\hline C11 & $0.6647(2)$ & $0.4210(2)$ & $0.1128(7)$ & $0.0416(11)$ \\
C12 & $0.7079(3)$ & $0.4721(2)$ & $0.0750(7)$ & $0.0485(13)$ \\
C13 & $0.7447(3)$ & $0.4762(3)$ & $-0.0845(8)$ & $0.0585(15)$ \\
H13 & 0.7729 & 0.5119 & -0.1054 & $0.070^{*}$ \\
C14 & $0.7386(3)$ & $0.4264(3)$ & $-0.2113(8)$ & $0.0633(15)$ \\
H14 & 0.7617 & 0.4286 & -0.3212 & $0.076^{*}$ \\
C15 & $0.6983(3)$ & $0.3734(3)$ & $-0.1744(7)$ & $0.0578(15)$ \\
H15 & 0.6958 & 0.3387 & -0.2576 & $0.069^{*}$ \\
C16 & $0.6612(3)$ & $0.3706(2)$ & $-0.0159(7)$ & $0.0494(13)$ \\
H16 & 0.6334 & 0.3344 & 0.0050 & $0.059^{*}$ \\
C111 & $0.6168(3)$ & $0.4224(3)$ & $0.2709(7)$ & $0.0491(13)$ \\
O111 & $0.5820(2)$ & $0.47044(17)$ & $0.3001(5)$ & $0.0719(11)$ \\
N111 & $0.6129(2)$ & $0.36582(19)$ & $0.3718(6)$ & $0.0468(12)$ \\
H111 & $0.642(2)$ & $0.339(2)$ & $0.348(7)$ & $0.056^{*}$ \\
N121 & $0.7207(2)$ & $0.5232(2)$ & $0.2121(8)$ & $0.0714(15)$ \\
O121 & $0.7199(3)$ & $0.5076(2)$ & $0.3713(7)$ & $0.1044(17)$ \\
O122 & $0.7336(2)$ & $0.57908(19)$ & $0.1580(7)$ & $0.1033(16)$ \\
S111 & $0.52655(6)$ & $0.42259(6)$ & $0.61418(19)$ & $0.0495(3)$ \\
C112 & $0.5731(2)$ & $0.3570(2)$ & $0.5239(6)$ & $0.0394(12)$
\end{tabular}




\begin{tabular}{|c|c|c|c|c|}
\hline N113 & $0.57191(18)$ & $0.30181(16)$ & $0.6124(6)$ & $0.0433(9)$ \\
\hline $\mathrm{C} 13 \mathrm{~A}$ & 0.5335 (2) & $0.3086(2)$ & $0.7691(7)$ & $0.0410(12)$ \\
\hline C114 & $0.5238(2)$ & $0.2610(2)$ & $0.9031(7)$ & $0.0498(13)$ \\
\hline H114 & 0.5421 & 0.2189 & 0.8892 & $0.060^{*}$ \\
\hline C115 & $0.4873(2)$ & $0.2755(3)$ & $1.0550(7)$ & $0.0549(15)$ \\
\hline H115 & 0.4815 & 0.2434 & 1.1447 & $0.066^{*}$ \\
\hline C116 & $0.4585(2)$ & $0.3382(2)$ & $1.0778(7)$ & $0.0512(13)$ \\
\hline C117 & $0.4667(2)$ & $0.3862(2)$ & $0.9472(7)$ & $0.0459(13)$ \\
\hline H117 & 0.4474 & 0.4278 & 0.9603 & $0.055^{*}$ \\
\hline C17A & $0.5048(2)$ & $0.3705(2)$ & 0.7939 (6) & $0.0421(12)$ \\
\hline O116 & $0.42262(18)$ & $0.34579(18)$ & $1.2344(5)$ & $0.0687(11)$ \\
\hline $\mathrm{C} 118$ & $0.3883(3)$ & $0.4068(3)$ & $1.2602(7)$ & $0.0763(18)$ \\
\hline H18A & 0.3605 & 0.4037 & 1.3665 & $0.115^{*}$ \\
\hline H18B & 0.3613 & 0.4159 & 1.1551 & $0.115^{*}$ \\
\hline $\mathrm{H} 18 \mathrm{C}$ & 0.4200 & 0.4419 & 1.2765 & $0.115^{*}$ \\
\hline $\mathrm{C} 21$ & $0.5587(2)$ & $0.10314(18)$ & $0.6179(7)$ & $0.0356(10)$ \\
\hline $\mathrm{C} 22$ & 0.4907 (2) & $0.09346(19)$ & $0.6067(7)$ & $0.0391(11)$ \\
\hline $\mathrm{C} 23$ & $0.4527(3)$ & $0.0724(2)$ & $0.7525(7)$ & 0.0475 (13) \\
\hline $\mathrm{H} 23$ & 0.4071 & 0.0658 & 0.7400 & $0.057^{*}$ \\
\hline $\mathrm{C} 24$ & $0.4839(3)$ & $0.0612(2)$ & $0.9165(7)$ & $0.0506(14)$ \\
\hline $\mathrm{H} 24$ & 0.4592 & 0.0472 & 1.0168 & $0.061^{*}$ \\
\hline $\mathrm{C} 25$ & $0.5511(3)$ & $0.0706(2)$ & $0.9332(7)$ & $0.0524(14)$ \\
\hline $\mathrm{H} 25$ & 0.5717 & 0.0631 & 1.0451 & $0.063^{*}$ \\
\hline $\mathrm{C} 26$ & $0.5889(3)$ & $0.0912(2)$ & $0.7851(6)$ & 0.0449 (13) \\
\hline $\mathrm{H} 26$ & 0.6346 & 0.0971 & 0.7979 & $0.054^{*}$ \\
\hline $\mathrm{C} 211$ & $0.6042(2)$ & $0.1170(2)$ & 0.4589 (6) & $0.0404(12)$ \\
\hline $\mathrm{O} 211$ & $0.61896(16)$ & $0.07405(15)$ & 0.3509 (4) & $0.0478(9)$ \\
\hline N211 & $0.6313(2)$ & 0.17844 (19) & $0.4542(5)$ & $0.0428(10)$ \\
\hline H211 & $0.617(2)$ & $0.210(2)$ & $0.522(5)$ & $0.051^{*}$ \\
\hline N221 & 0.4558 (2) & $0.1058(2)$ & $0.4325(6)$ & $0.0519(11)$ \\
\hline $\mathrm{O} 221$ & $0.48494(19)$ & $0.13925(18)$ & $0.3175(5)$ & $0.0705(12)$ \\
\hline $\mathrm{O} 222$ & $0.4004(2)$ & $0.0816(2)$ & $0.4107(6)$ & $0.0818(13)$ \\
\hline S211 & $0.70172(6)$ & $0.15015(5)$ & $0.14183(17)$ & $0.0474(3)$ \\
\hline C212 & 0.6797 (2) & 0.1980 (2) & $0.3302(6)$ & $0.0372(12)$ \\
\hline N213 & $0.71002(18)$ & $0.25434(17)$ & $0.3486(5)$ & $0.0384(9)$ \\
\hline $\mathrm{C} 23 \mathrm{~A}$ & $0.7553(2)$ & $0.2629(2)$ & $0.2067(6)$ & $0.0375(12)$ \\
\hline $\mathrm{C} 214$ & $0.7983(2)$ & $0.3165(2)$ & $0.1862(6)$ & $0.0449(13)$ \\
\hline H214 & 0.8000 & 0.3498 & 0.2739 & $0.054^{*}$ \\
\hline C215 & $0.8381(2)$ & $0.3192(2)$ & $0.0346(7)$ & $0.0488(14)$ \\
\hline $\mathrm{H} 215$ & 0.8683 & 0.3539 & 0.0229 & $0.059^{*}$ \\
\hline $\mathrm{C} 216$ & $0.8344(2)$ & $0.2713(2)$ & $-0.1031(7)$ & $0.0468(13)$ \\
\hline $\mathrm{C} 217$ & 0.7948 (2) & $0.2163(2)$ & $-0.0808(6)$ & $0.0463(13)$ \\
\hline $\mathrm{H} 217$ & 0.7942 & 0.1825 & -0.1671 & $0.056^{*}$ \\
\hline $\mathrm{C} 27 \mathrm{~A}$ & $0.7560(2)$ & $0.2131(2)$ & $0.0749(6)$ & $0.0393(12)$ \\
\hline $\mathrm{O} 216$ & $0.87332(17)$ & $0.28389(17)$ & $-0.2525(5)$ & $0.0614(10)$ \\
\hline $\mathrm{C} 218$ & $0.8728(3)$ & $0.2358(3)$ & $-0.3963(7)$ & $0.0652(15)$ \\
\hline $\mathrm{H} 28 \mathrm{~A}$ & 0.9029 & 0.2494 & -0.4911 & $0.098^{*}$ \\
\hline H28B & 0.8286 & 0.2321 & -0.4453 & $0.098^{*}$ \\
\hline
\end{tabular}




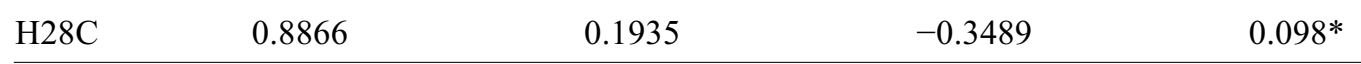

Atomic displacement parameters $\left(\AA^{2}\right)$

\begin{tabular}{|c|c|c|c|c|c|c|}
\hline & $U^{11}$ & $U^{22}$ & $U^{33}$ & $U^{12}$ & $U^{13}$ & $U^{23}$ \\
\hline $\mathrm{C} 11$ & $0.047(3)$ & $0.038(2)$ & $0.040(3)$ & $0.000(2)$ & $-0.002(3)$ & 0.007 (3) \\
\hline $\mathrm{C} 12$ & $0.058(3)$ & $0.039(3)$ & $0.049(3)$ & $0.000(3)$ & -0.004 & $0.003(2)$ \\
\hline C13 & $0.055(4)$ & $0.055(4)$ & $0.066(4)$ & $-0.004(3)$ & $0.005(3)$ & $0.013(3)$ \\
\hline $\mathrm{C} 14$ & $0.061(4)$ & $0.082(4)$ & $0.048(3)$ & $0.001(4)$ & $0.002(3)$ & $0.010(3)$ \\
\hline $\mathrm{C} 15$ & $0.058(4)$ & 0.069 (4) & $0.047(3)$ & $0.002(3)$ & -0.008 & -0.009 (3) \\
\hline $\mathrm{C} 16$ & $0.046(3)$ & $0.050(3)$ & $0.053(3)$ & $0.001(3)$ & $-0.011(3)$ & $-0.002(3)$ \\
\hline C111 & $0.054(4)$ & $0.044(3)$ & $0.049(3)$ & $-0.003(3)$ & $0.001(3)$ & $0.002(3)$ \\
\hline O111 & $0.097(3)$ & $0.048(2)$ & $0.071(3)$ & $0.026(2)$ & $0.021(2)$ & 0.0119 (19) \\
\hline N111 & $0.052(3)$ & 0.035 & $0.054(3)$ & $0.006(2)$ & $0.007(2)$ & $0.000(2)$ \\
\hline N121 & $0.083(4)$ & 0.050 & $0.081(4)$ & -0.005 & $0.001(3)$ & $-0.006(3)$ \\
\hline O121 & $0.159(5)$ & $0.088(4)$ & $0.066(3)$ & $-0.017(3)$ & -0.020 & $-0.016(3)$ \\
\hline $\mathrm{O} 122$ & $0.136(4)$ & $0.047(2)$ & $0.127(4)$ & $-0.024(3)$ & $0.019(3)$ & $-0.014(3)$ \\
\hline S111 & $0.0575(8)$ & $0.0389(6)$ & $0.0519(8)$ & $0.0095(6)$ & $0.0048(8)$ & $0.0053(7)$ \\
\hline $\mathrm{C} 112$ & $0.036(3)$ & $0.037(3)$ & $0.045(3)$ & $-0.001(2)$ & $0.001(2)$ & $-0.004(2)$ \\
\hline N113 & $0.048(2)$ & $0.030(2)$ & $0.052(2)$ & $-0.0026(18)$ & $0.003(2)$ & $0.004(2)$ \\
\hline C13A & $0.039(3)$ & $0.034(3)$ & $0.050(3)$ & $-0.004(2)$ & $0.000(3)$ & $0.000(2)$ \\
\hline C114 & $0.055(4)$ & $0.032(3)$ & $0.062(4)$ & $0.001(3)$ & $0.003(3)$ & $0.003(3)$ \\
\hline C115 & $0.059(4)$ & $0.045(3)$ & $0.060(4)$ & $-0.007(3)$ & $0.008(3)$ & 0.013 (2) \\
\hline C116 & $0.053(3)$ & $0.043(3)$ & $0.057(4)$ & -0.003 & -0.001 & $-0.003(3)$ \\
\hline C117 & $0.051(3)$ & $0.036(3)$ & $0.051(3)$ & $0.002(3)$ & $0.003(3)$ & $0.003(2)$ \\
\hline C17A & $0.043(3)$ & $0.033(3)$ & $0.050(3)$ & $-0.003(2)$ & -0.005 & $0.003(2)$ \\
\hline O116 & $0.087(3)$ & $0.060(3)$ & $0.060(2)$ & 0.007 (2) & $0.028(2)$ & $0.008(2)$ \\
\hline C118 & $0.087(5)$ & $0.071(4)$ & $0.071(4)$ & $0.014(4)$ & $0.027(4)$ & $-0.002(3)$ \\
\hline $\mathrm{C} 21$ & $0.037(3)$ & $0.030(2)$ & $0.040(3)$ & $-0.007(2)$ & $0.002(3)$ & $-0.002(2)$ \\
\hline $\mathrm{C} 22$ & $0.048(3)$ & $0.034(3)$ & $0.035(3)$ & $0.006(2)$ & $0.002(3)$ & $0.005(2)$ \\
\hline $\mathrm{C} 23$ & $0.041(3)$ & $0.045(3)$ & $0.056(4)$ & $0.004(3)$ & $0.006(3)$ & -0.001 \\
\hline $\mathrm{C} 24$ & $0.062(4)$ & $0.045(3)$ & $0.045(3)$ & $0.000(3)$ & $0.013(3)$ & $0.006(2)$ \\
\hline $\mathrm{C} 25$ & $0.071(4)$ & $0.046(3)$ & $0.040(3)$ & $-0.004(3)$ & -0.009 (3) & $0.001(3)$ \\
\hline $\mathrm{C} 26$ & $0.043(3)$ & $0.050(3)$ & $0.042(3)$ & $-0.005(3)$ & $-0.008(3)$ & $-0.003(3)$ \\
\hline $\mathrm{C} 211$ & $0.043(3)$ & $0.038(3)$ & $0.040(3)$ & $-0.002(3)$ & $-0.006(3)$ & $0.002(2)$ \\
\hline $\mathrm{O} 211$ & $0.060(2)$ & $0.0379(18)$ & $0.045(2)$ & $-0.0039(17)$ & $0.0096(18)$ & $-0.0089(15)$ \\
\hline N211 & $0.051(3)$ & $0.035(2)$ & $0.043(3)$ & $-0.004(2)$ & $0.009(2)$ & $-0.0040(19)$ \\
\hline N221 & $0.052(3)$ & $0.047(3)$ & $0.057(3)$ & $0.010(2)$ & $-0.012(3)$ & $0.001(2)$ \\
\hline $\mathrm{O} 221$ & $0.077(3)$ & $0.080(3)$ & $0.054(2)$ & $0.005(2)$ & $-0.007(2)$ & 0.027 (2) \\
\hline $\mathrm{O} 222$ & $0.058(3)$ & $0.092(3)$ & $0.096(3)$ & $-0.005(3)$ & $-0.035(2)$ & $0.017(2)$ \\
\hline $\mathrm{S} 211$ & $0.0530(8)$ & $0.0404(7)$ & $0.0487(8)$ & $-0.0104(6)$ & $0.0091(7)$ & $-0.0073(7)$ \\
\hline $\mathrm{C} 212$ & $0.041(3)$ & $0.032(3)$ & $0.039(3)$ & $-0.002(2)$ & $0.002(2)$ & $0.002(2)$ \\
\hline N213 & $0.037(2)$ & $0.034(2)$ & $0.044(2)$ & $-0.0061(19)$ & $0.008(2)$ & $-0.0021(18)$ \\
\hline $\mathrm{C} 23 \mathrm{~A}$ & $0.035(3)$ & $0.032(3)$ & $0.045(3)$ & $-0.002(2)$ & $-0.006(2)$ & $-0.003(2)$ \\
\hline $\mathrm{C} 214$ & $0.041(3)$ & $0.045(3)$ & $0.049(3)$ & -0.003 & $0.004(3)$ & $-0.005(2)$ \\
\hline $\mathrm{C} 215$ & $0.043(3)$ & $0.048(3)$ & $0.055(4)$ & $-0.014(3)$ & $-0.004(3)$ & $0.005(3)$ \\
\hline $\mathrm{C} 216$ & $0.039(3)$ & $0.056(3)$ & $0.046(3)$ & -0.003 & 0.007 (3) & 0.007 (3) \\
\hline $\mathrm{C} 217$ & $0.051(3)$ & $0.043(3)$ & $0.045(3)$ & $-0.003(3)$ & $0.003(3)$ & $-0.005(2)$ \\
\hline
\end{tabular}




\begin{tabular}{lllllll}
$\mathrm{C} 27 \mathrm{~A}$ & $0.034(3)$ & $0.037(3)$ & $0.047(3)$ & $-0.006(2)$ & $0.004(2)$ & $0.002(2)$ \\
$\mathrm{O} 216$ & $0.063(3)$ & $0.068(3)$ & $0.053(2)$ & $-0.017(2)$ & $0.012(2)$ & $0.0009(19)$ \\
$\mathrm{C} 218$ & $0.058(4)$ & $0.088(4)$ & $0.050(3)$ & $-0.010(3)$ & $0.011(3)$ & $0.002(3)$ \\
\hline
\end{tabular}

Geometric parameters $\left(\AA,{ }^{\circ}\right)$

\begin{tabular}{|c|c|c|c|}
\hline $\mathrm{C} 11-\mathrm{C} 12$ & $1.374(6)$ & $\mathrm{C} 21-\mathrm{C} 22$ & $1.382(5)$ \\
\hline $\mathrm{C} 11-\mathrm{C} 16$ & $1.389(6)$ & $\mathrm{C} 21-\mathrm{C} 26$ & $1.388(6)$ \\
\hline $\mathrm{C} 11-\mathrm{C} 111$ & $1.507(7)$ & $\mathrm{C} 21-\mathrm{C} 211$ & $1.507(6)$ \\
\hline $\mathrm{C} 12-\mathrm{C} 13$ & $1.384(6)$ & $\mathrm{C} 22-\mathrm{C} 23$ & $1.379(6)$ \\
\hline $\mathrm{C} 12-\mathrm{N} 121$ & $1.461(6)$ & $\mathrm{C} 22-\mathrm{N} 221$ & $1.477(6)$ \\
\hline $\mathrm{C} 13-\mathrm{C} 14$ & $1.373(7)$ & $\mathrm{C} 23-\mathrm{C} 24$ & $1.373(6)$ \\
\hline $\mathrm{C} 13-\mathrm{H} 13$ & 0.9300 & $\mathrm{C} 23-\mathrm{H} 23$ & 0.9300 \\
\hline $\mathrm{C} 14-\mathrm{C} 15$ & $1.367(7)$ & $\mathrm{C} 24-\mathrm{C} 25$ & $1.370(6)$ \\
\hline C14-H14 & 0.9300 & $\mathrm{C} 24-\mathrm{H} 24$ & 0.9300 \\
\hline $\mathrm{C} 15-\mathrm{C} 16$ & $1.381(7)$ & $\mathrm{C} 25-\mathrm{C} 26$ & $1.387(6)$ \\
\hline $\mathrm{C} 15-\mathrm{H} 15$ & 0.9300 & $\mathrm{C} 25-\mathrm{H} 25$ & 0.9300 \\
\hline C16-H16 & 0.9300 & $\mathrm{C} 26-\mathrm{H} 26$ & 0.9300 \\
\hline $\mathrm{C} 111-\mathrm{O} 111$ & $1.214(5)$ & $\mathrm{C} 211-\mathrm{O} 211$ & $1.209(5)$ \\
\hline C111-N111 & $1.361(6)$ & $\mathrm{C} 211-\mathrm{N} 211$ & $1.354(5)$ \\
\hline $\mathrm{N} 111-\mathrm{C} 112$ & $1.383(6)$ & $\mathrm{N} 211-\mathrm{C} 212$ & $1.388(6)$ \\
\hline N111-H111 & $0.81(4)$ & $\mathrm{N} 211-\mathrm{H} 211$ & $0.86(4)$ \\
\hline $\mathrm{N} 121-\mathrm{O} 121$ & $1.207(6)$ & $\mathrm{N} 221-\mathrm{O} 222$ & $1.225(5)$ \\
\hline $\mathrm{N} 121-\mathrm{O} 122$ & $1.223(5)$ & $\mathrm{N} 221-\mathrm{O} 221$ & $1.228(5)$ \\
\hline $\mathrm{S} 111-\mathrm{C} 17 \mathrm{~A}$ & $1.739(5)$ & $\mathrm{S} 211-\mathrm{C} 212$ & $1.740(4)$ \\
\hline $\mathrm{S} 111-\mathrm{C} 112$ & $1.749(5)$ & S211-C27A & $1.744(4)$ \\
\hline C112-N113 & $1.288(5)$ & $\mathrm{C} 212-\mathrm{N} 213$ & $1.296(5)$ \\
\hline $\mathrm{N} 113-\mathrm{C} 13 \mathrm{~A}$ & $1.389(6)$ & $\mathrm{N} 213-\mathrm{C} 23 \mathrm{~A}$ & $1.391(5)$ \\
\hline $\mathrm{C} 13 \mathrm{~A}-\mathrm{C} 17 \mathrm{~A}$ & $1.387(5)$ & $\mathrm{C} 23 \mathrm{~A}-\mathrm{C} 27 \mathrm{~A}$ & $1.392(6)$ \\
\hline $\mathrm{C} 13 \mathrm{~A}-\mathrm{C} 114$ & $1.387(6)$ & $\mathrm{C} 23 \mathrm{~A}-\mathrm{C} 214$ & $1.393(6)$ \\
\hline $\mathrm{C} 114-\mathrm{C} 115$ & $1.364(6)$ & $\mathrm{C} 214-\mathrm{C} 215$ & $1.368(6)$ \\
\hline C114-H114 & 0.9300 & $\mathrm{C} 214-\mathrm{H} 214$ & 0.9300 \\
\hline $\mathrm{C} 115-\mathrm{C} 116$ & $1.400(6)$ & $\mathrm{C} 215-\mathrm{C} 216$ & $1.399(6)$ \\
\hline $\mathrm{C} 115-\mathrm{H} 115$ & 0.9300 & $\mathrm{C} 215-\mathrm{H} 215$ & 0.9300 \\
\hline $\mathrm{C} 116-\mathrm{O} 116$ & $1.364(6)$ & $\mathrm{C} 216-\mathrm{O} 216$ & $1.368(5)$ \\
\hline $\mathrm{C} 116-\mathrm{C} 117$ & $1.370(6)$ & $\mathrm{C} 216-\mathrm{C} 217$ & $1.374(6)$ \\
\hline $\mathrm{C} 117-\mathrm{C} 17 \mathrm{~A}$ & $1.395(6)$ & $\mathrm{C} 217-\mathrm{C} 27 \mathrm{~A}$ & $1.383(6)$ \\
\hline C117-H117 & 0.9300 & $\mathrm{C} 217-\mathrm{H} 217$ & 0.9300 \\
\hline $\mathrm{O} 116-\mathrm{C} 118$ & $1.422(5)$ & $\mathrm{O} 216-\mathrm{C} 218$ & $1.432(6)$ \\
\hline $\mathrm{C} 118-\mathrm{H} 18 \mathrm{~A}$ & 0.9600 & $\mathrm{C} 218-\mathrm{H} 28 \mathrm{~A}$ & 0.9600 \\
\hline $\mathrm{C} 118-\mathrm{H} 18 \mathrm{~B}$ & 0.9600 & $\mathrm{C} 218-\mathrm{H} 28 \mathrm{~B}$ & 0.9600 \\
\hline $\mathrm{C} 118-\mathrm{H} 18 \mathrm{C}$ & 0.9600 & $\mathrm{C} 218-\mathrm{H} 28 \mathrm{C}$ & 0.9600 \\
\hline $\mathrm{C} 12-\mathrm{C} 11-\mathrm{C} 16$ & $116.4(5)$ & $\mathrm{C} 22-\mathrm{C} 21-\mathrm{C} 26$ & $117.4(4)$ \\
\hline $\mathrm{C} 12-\mathrm{C} 11-\mathrm{C} 111$ & $123.0(5)$ & $\mathrm{C} 22-\mathrm{C} 21-\mathrm{C} 211$ & $125.5(4)$ \\
\hline $\mathrm{C} 16-\mathrm{C} 11-\mathrm{C} 111$ & $120.1(4)$ & $\mathrm{C} 26-\mathrm{C} 21-\mathrm{C} 211$ & $116.6(4)$ \\
\hline $\mathrm{C} 11-\mathrm{C} 12-\mathrm{C} 13$ & $123.4(5)$ & $\mathrm{C} 23-\mathrm{C} 22-\mathrm{C} 21$ & $123.0(5)$ \\
\hline $\mathrm{C} 11-\mathrm{C} 12-\mathrm{N} 121$ & $120.1(5)$ & $\mathrm{C} 23-\mathrm{C} 22-\mathrm{N} 221$ & $117.3(4)$ \\
\hline
\end{tabular}




\begin{tabular}{|c|c|c|c|}
\hline $\mathrm{C} 13-\mathrm{C} 12-\mathrm{N} 121$ & $116.3(5)$ & $\mathrm{C} 21-\mathrm{C} 22-\mathrm{N} 221$ & $119.7(4)$ \\
\hline $\mathrm{C} 14-\mathrm{C} 13-\mathrm{C} 12$ & $118.7(5)$ & $\mathrm{C} 24-\mathrm{C} 23-\mathrm{C} 22$ & $118.4(5)$ \\
\hline $\mathrm{C} 14-\mathrm{C} 13-\mathrm{H} 13$ & 120.7 & $\mathrm{C} 24-\mathrm{C} 23-\mathrm{H} 23$ & 120.8 \\
\hline $\mathrm{C} 12-\mathrm{C} 13-\mathrm{H} 13$ & 120.7 & $\mathrm{C} 22-\mathrm{C} 23-\mathrm{H} 23$ & 120.8 \\
\hline $\mathrm{C} 15-\mathrm{C} 14-\mathrm{C} 13$ & $119.3(5)$ & $\mathrm{C} 25-\mathrm{C} 24-\mathrm{C} 23$ & $120.3(5)$ \\
\hline $\mathrm{C} 15-\mathrm{C} 14-\mathrm{H} 14$ & 120.3 & $\mathrm{C} 25-\mathrm{C} 24-\mathrm{H} 24$ & 119.8 \\
\hline $\mathrm{C} 13-\mathrm{C} 14-\mathrm{H} 14$ & 120.3 & $\mathrm{C} 23-\mathrm{C} 24-\mathrm{H} 24$ & 119.8 \\
\hline $\mathrm{C} 14-\mathrm{C} 15-\mathrm{C} 16$ & $121.3(5)$ & $\mathrm{C} 24-\mathrm{C} 25-\mathrm{C} 26$ & $120.8(5)$ \\
\hline $\mathrm{C} 14-\mathrm{C} 15-\mathrm{H} 15$ & 119.4 & $\mathrm{C} 24-\mathrm{C} 25-\mathrm{H} 25$ & 119.6 \\
\hline $\mathrm{C} 16-\mathrm{C} 15-\mathrm{H} 15$ & 119.4 & $\mathrm{C} 26-\mathrm{C} 25-\mathrm{H} 25$ & 119.6 \\
\hline $\mathrm{C} 15-\mathrm{C} 16-\mathrm{C} 11$ & $120.8(5)$ & $\mathrm{C} 25-\mathrm{C} 26-\mathrm{C} 21$ & $120.1(5)$ \\
\hline $\mathrm{C} 15-\mathrm{C} 16-\mathrm{H} 16$ & 119.6 & $\mathrm{C} 25-\mathrm{C} 26-\mathrm{H} 26$ & 119.9 \\
\hline $\mathrm{C} 11-\mathrm{C} 16-\mathrm{H} 16$ & 119.6 & $\mathrm{C} 21-\mathrm{C} 26-\mathrm{H} 26$ & 119.9 \\
\hline $\mathrm{O} 111-\mathrm{C} 111-\mathrm{N} 111$ & $122.7(5)$ & $\mathrm{O} 211-\mathrm{C} 211-\mathrm{N} 211$ & $122.7(5)$ \\
\hline $\mathrm{O} 111-\mathrm{C} 111-\mathrm{C} 11$ & $121.2(5)$ & $\mathrm{O} 211-\mathrm{C} 211-\mathrm{C} 21$ & $121.4(4)$ \\
\hline $\mathrm{N} 111-\mathrm{C} 111-\mathrm{C} 11$ & $116.0(5)$ & $\mathrm{N} 211-\mathrm{C} 211-\mathrm{C} 21$ & $115.6(4)$ \\
\hline $\mathrm{C} 111-\mathrm{N} 111-\mathrm{C} 112$ & $125.3(4)$ & $\mathrm{C} 211-\mathrm{N} 211-\mathrm{C} 212$ & $123.9(4)$ \\
\hline C111-N111-H111 & $113(4)$ & $\mathrm{C} 211-\mathrm{N} 211-\mathrm{H} 211$ & $122(3)$ \\
\hline C112-N111-H111 & $121(4)$ & $\mathrm{C} 212-\mathrm{N} 211-\mathrm{H} 211$ & $114(3)$ \\
\hline $\mathrm{O} 121-\mathrm{N} 121-\mathrm{O} 122$ & $123.8(5)$ & $\mathrm{O} 222-\mathrm{N} 221-\mathrm{O} 221$ & $124.2(5)$ \\
\hline $\mathrm{O} 121-\mathrm{N} 121-\mathrm{C} 12$ & $118.5(5)$ & $\mathrm{O} 222-\mathrm{N} 221-\mathrm{C} 22$ & $118.5(5)$ \\
\hline $\mathrm{O} 122-\mathrm{N} 121-\mathrm{C} 12$ & $117.7(5)$ & $\mathrm{O} 221-\mathrm{N} 221-\mathrm{C} 22$ & $117.3(4)$ \\
\hline $\mathrm{C} 17 \mathrm{~A}-\mathrm{S} 111-\mathrm{C} 112$ & $87.9(2)$ & $\mathrm{C} 212-\mathrm{S} 211-\mathrm{C} 27 \mathrm{~A}$ & $88.7(2)$ \\
\hline N113-C112-N111 & $121.8(4)$ & $\mathrm{N} 213-\mathrm{C} 212-\mathrm{N} 211$ & $120.7(4)$ \\
\hline $\mathrm{N} 113-\mathrm{C} 112-\mathrm{S} 111$ & $117.0(4)$ & $\mathrm{N} 213-\mathrm{C} 212-\mathrm{S} 211$ & $116.7(3)$ \\
\hline $\mathrm{N} 111-\mathrm{C} 112-\mathrm{S} 111$ & $121.1(4)$ & $\mathrm{N} 211-\mathrm{C} 212-\mathrm{S} 211$ & $122.6(3)$ \\
\hline $\mathrm{C} 112-\mathrm{N} 113-\mathrm{C} 13 \mathrm{~A}$ & $109.9(4)$ & $\mathrm{C} 212-\mathrm{N} 213-\mathrm{C} 23 \mathrm{~A}$ & $109.7(4)$ \\
\hline $\mathrm{C} 17 \mathrm{~A}-\mathrm{C} 13 \mathrm{~A}-\mathrm{C} 114$ & $118.2(4)$ & $\mathrm{N} 213-\mathrm{C} 23 \mathrm{~A}-\mathrm{C} 27 \mathrm{~A}$ & $115.8(4)$ \\
\hline $\mathrm{C} 17 \mathrm{~A}-\mathrm{C} 13 \mathrm{~A}-\mathrm{N} 113$ & $115.3(4)$ & $\mathrm{N} 213-\mathrm{C} 23 \mathrm{~A}-\mathrm{C} 214$ & $125.6(4)$ \\
\hline $\mathrm{C} 114-\mathrm{C} 13 \mathrm{~A}-\mathrm{N} 113$ & $126.4(4)$ & $\mathrm{C} 27 \mathrm{~A}-\mathrm{C} 23 \mathrm{~A}-\mathrm{C} 214$ & $118.6(4)$ \\
\hline $\mathrm{C} 115-\mathrm{C} 114-\mathrm{C} 13 \mathrm{~A}$ & $120.2(5)$ & $\mathrm{C} 215-\mathrm{C} 214-\mathrm{C} 23 \mathrm{~A}$ & $118.8(4)$ \\
\hline $\mathrm{C} 115-\mathrm{C} 114-\mathrm{H} 114$ & 119.9 & $\mathrm{C} 215-\mathrm{C} 214-\mathrm{H} 214$ & 120.6 \\
\hline $\mathrm{C} 13 \mathrm{~A}-\mathrm{C} 114-\mathrm{H} 114$ & 119.9 & $\mathrm{C} 23 \mathrm{~A}-\mathrm{C} 214-\mathrm{H} 214$ & 120.6 \\
\hline $\mathrm{C} 114-\mathrm{C} 115-\mathrm{C} 116$ & $120.9(5)$ & $\mathrm{C} 214-\mathrm{C} 215-\mathrm{C} 216$ & $121.8(5)$ \\
\hline $\mathrm{C} 114-\mathrm{C} 115-\mathrm{H} 115$ & 119.6 & $\mathrm{C} 214-\mathrm{C} 215-\mathrm{H} 215$ & 119.1 \\
\hline $\mathrm{C} 116-\mathrm{C} 115-\mathrm{H} 115$ & 119.6 & $\mathrm{C} 216-\mathrm{C} 215-\mathrm{H} 215$ & 119.1 \\
\hline $\mathrm{O} 116-\mathrm{C} 116-\mathrm{C} 117$ & $124.8(5)$ & $\mathrm{O} 216-\mathrm{C} 216-\mathrm{C} 217$ & $125.1(4)$ \\
\hline $\mathrm{O} 116-\mathrm{C} 116-\mathrm{C} 115$ & $114.8(5)$ & $\mathrm{O} 216-\mathrm{C} 216-\mathrm{C} 215$ & $114.7(4)$ \\
\hline $\mathrm{C} 117-\mathrm{C} 116-\mathrm{C} 115$ & $120.3(5)$ & $\mathrm{C} 217-\mathrm{C} 216-\mathrm{C} 215$ & $120.1(4)$ \\
\hline $\mathrm{C} 116-\mathrm{C} 117-\mathrm{C} 17 \mathrm{~A}$ & $117.9(4)$ & $\mathrm{C} 216-\mathrm{C} 217-\mathrm{C} 27 \mathrm{~A}$ & $117.5(4)$ \\
\hline $\mathrm{C} 116-\mathrm{C} 117-\mathrm{H} 117$ & 121.1 & $\mathrm{C} 216-\mathrm{C} 217-\mathrm{H} 217$ & 121.2 \\
\hline $\mathrm{C} 17 \mathrm{~A}-\mathrm{C} 117-\mathrm{H} 117$ & 121.1 & $\mathrm{C} 27 \mathrm{~A}-\mathrm{C} 217-\mathrm{H} 217$ & 121.2 \\
\hline $\mathrm{C} 13 \mathrm{~A}-\mathrm{C} 17 \mathrm{~A}-\mathrm{C} 117$ & $122.5(4)$ & $\mathrm{C} 217-\mathrm{C} 27 \mathrm{~A}-\mathrm{C} 23 \mathrm{~A}$ & $122.9(4)$ \\
\hline $\mathrm{C} 13 \mathrm{~A}-\mathrm{C} 17 \mathrm{~A}-\mathrm{S} 111$ & $109.9(4)$ & $\mathrm{C} 217-\mathrm{C} 27 \mathrm{~A}-\mathrm{S} 211$ & $128.2(4)$ \\
\hline $\mathrm{C} 117-\mathrm{C} 17 \mathrm{~A}-\mathrm{S} 111$ & $127.6(4)$ & $\mathrm{C} 23 \mathrm{~A}-\mathrm{C} 27 \mathrm{~A}-\mathrm{S} 211$ & $108.9(3)$ \\
\hline $\mathrm{C} 116-\mathrm{O} 116-\mathrm{C} 118$ & $117.7(4)$ & $\mathrm{C} 216-\mathrm{O} 216-\mathrm{C} 218$ & $117.3(4)$ \\
\hline $\mathrm{O} 116-\mathrm{C} 118-\mathrm{H} 18 \mathrm{~A}$ & 109.5 & $\mathrm{O} 216-\mathrm{C} 218-\mathrm{H} 28 \mathrm{~A}$ & 109.5 \\
\hline $\mathrm{O} 116-\mathrm{C} 118-\mathrm{H} 18 \mathrm{~B}$ & 109.5 & $\mathrm{O} 216-\mathrm{C} 218-\mathrm{H} 28 \mathrm{~B}$ & 109.5 \\
\hline
\end{tabular}




\begin{tabular}{|c|c|c|c|}
\hline $\mathrm{H} 18 \mathrm{~A}-\mathrm{C} 118-\mathrm{H} 18 \mathrm{~B}$ & 109.5 & $\mathrm{H} 28 \mathrm{~A}-\mathrm{C} 218-\mathrm{H} 28 \mathrm{~B}$ & 109.5 \\
\hline $\mathrm{O} 116-\mathrm{C} 118-\mathrm{H} 18 \mathrm{C}$ & 109.5 & $\mathrm{O} 216-\mathrm{C} 218-\mathrm{H} 28 \mathrm{C}$ & 109.5 \\
\hline $\mathrm{H} 18 \mathrm{~A}-\mathrm{C} 118-\mathrm{H} 18 \mathrm{C}$ & 109.5 & $\mathrm{H} 28 \mathrm{~A}-\mathrm{C} 218-\mathrm{H} 28 \mathrm{C}$ & 109.5 \\
\hline $\mathrm{H} 18 \mathrm{~B}-\mathrm{C} 118-\mathrm{H} 18 \mathrm{C}$ & 109.5 & $\mathrm{H} 28 \mathrm{~B}-\mathrm{C} 218-\mathrm{H} 28 \mathrm{C}$ & 109.5 \\
\hline $\mathrm{C} 16-\mathrm{C} 11-\mathrm{C} 12-\mathrm{C} 13$ & $2.6(7)$ & $\mathrm{C} 26-\mathrm{C} 21-\mathrm{C} 22-\mathrm{C} 23$ & $-0.5(6)$ \\
\hline $\mathrm{C} 111-\mathrm{C} 11-\mathrm{C} 12-\mathrm{C} 13$ & $-169.6(5)$ & $\mathrm{C} 211-\mathrm{C} 21-\mathrm{C} 22-\mathrm{C} 23$ & $171.1(4)$ \\
\hline $\mathrm{C} 16-\mathrm{C} 11-\mathrm{C} 12-\mathrm{N} 121$ & $-172.8(4)$ & $\mathrm{C} 26-\mathrm{C} 21-\mathrm{C} 22-\mathrm{N} 221$ & $179.6(4)$ \\
\hline $\mathrm{C} 111-\mathrm{C} 11-\mathrm{C} 12-\mathrm{N} 121$ & $15.0(7)$ & $\mathrm{C} 211-\mathrm{C} 21-\mathrm{C} 22-\mathrm{N} 221$ & $-8.8(6)$ \\
\hline $\mathrm{C} 11-\mathrm{C} 12-\mathrm{C} 13-\mathrm{C} 14$ & $-0.9(8)$ & $\mathrm{C} 21-\mathrm{C} 22-\mathrm{C} 23-\mathrm{C} 24$ & $0.7(7)$ \\
\hline $\mathrm{N} 121-\mathrm{C} 12-\mathrm{C} 13-\mathrm{C} 14$ & $174.7(5)$ & $\mathrm{N} 221-\mathrm{C} 22-\mathrm{C} 23-\mathrm{C} 24$ & $-179.3(4)$ \\
\hline $\mathrm{C} 12-\mathrm{C} 13-\mathrm{C} 14-\mathrm{C} 15$ & $-2.0(8)$ & $\mathrm{C} 22-\mathrm{C} 23-\mathrm{C} 24-\mathrm{C} 25$ & $-0.3(7)$ \\
\hline $\mathrm{C} 13-\mathrm{C} 14-\mathrm{C} 15-\mathrm{C} 16$ & $3.1(8)$ & $\mathrm{C} 23-\mathrm{C} 24-\mathrm{C} 25-\mathrm{C} 26$ & $-0.3(7)$ \\
\hline $\mathrm{C} 14-\mathrm{C} 15-\mathrm{C} 16-\mathrm{C} 11$ & $-1.3(8)$ & $\mathrm{C} 24-\mathrm{C} 25-\mathrm{C} 26-\mathrm{C} 21$ & $0.6(7)$ \\
\hline $\mathrm{C} 12-\mathrm{C} 11-\mathrm{C} 16-\mathrm{C} 15$ & $-1.5(7)$ & $\mathrm{C} 22-\mathrm{C} 21-\mathrm{C} 26-\mathrm{C} 25$ & $-0.2(6)$ \\
\hline $\mathrm{C} 111-\mathrm{C} 11-\mathrm{C} 16-\mathrm{C} 15$ & $170.9(4)$ & $\mathrm{C} 211-\mathrm{C} 21-\mathrm{C} 26-\mathrm{C} 25$ & $-172.6(4)$ \\
\hline $\mathrm{C} 12-\mathrm{C} 11-\mathrm{C} 111-\mathrm{O} 111$ & $47.8(7)$ & $\mathrm{C} 22-\mathrm{C} 21-\mathrm{C} 211-\mathrm{O} 211$ & $-73.2(6)$ \\
\hline $\mathrm{C} 16-\mathrm{C} 11-\mathrm{C} 111-\mathrm{O} 111$ & $-124.1(5)$ & $\mathrm{C} 26-\mathrm{C} 21-\mathrm{C} 211-\mathrm{O} 211$ & $98.5(5)$ \\
\hline $\mathrm{C} 12-\mathrm{C} 11-\mathrm{C} 111-\mathrm{N} 111$ & $-135.1(5)$ & $\mathrm{C} 22-\mathrm{C} 21-\mathrm{C} 211-\mathrm{N} 211$ & $111.9(5)$ \\
\hline $\mathrm{C} 16-\mathrm{C} 11-\mathrm{C} 111-\mathrm{N} 111$ & $53.0(7)$ & $\mathrm{C} 26-\mathrm{C} 21-\mathrm{C} 211-\mathrm{N} 211$ & $-76.5(5)$ \\
\hline $\mathrm{O} 111-\mathrm{C} 111-\mathrm{N} 111-\mathrm{C} 112$ & $-4.1(8)$ & $\mathrm{O} 211-\mathrm{C} 211-\mathrm{N} 211-\mathrm{C} 212$ & $-2.0(7)$ \\
\hline $\mathrm{C} 11-\mathrm{C} 111-\mathrm{N} 111-\mathrm{C} 112$ & $178.8(4)$ & $\mathrm{C} 21-\mathrm{C} 211-\mathrm{N} 211-\mathrm{C} 212$ & $172.8(4)$ \\
\hline $\mathrm{C} 11-\mathrm{C} 12-\mathrm{N} 121-\mathrm{O} 121$ & $33.1(8)$ & $\mathrm{C} 23-\mathrm{C} 22-\mathrm{N} 221-\mathrm{O} 222$ & $-18.2(6)$ \\
\hline $\mathrm{C} 13-\mathrm{C} 12-\mathrm{N} 121-\mathrm{O} 121$ & $-142.7(6)$ & $\mathrm{C} 21-\mathrm{C} 22-\mathrm{N} 221-\mathrm{O} 222$ & $161.7(4)$ \\
\hline $\mathrm{C} 11-\mathrm{C} 12-\mathrm{N} 121-\mathrm{O} 122$ & $-148.9(5)$ & $\mathrm{C} 23-\mathrm{C} 22-\mathrm{N} 221-\mathrm{O} 221$ & $162.7(4)$ \\
\hline $\mathrm{C} 13-\mathrm{C} 12-\mathrm{N} 121-\mathrm{O} 122$ & $35.4(7)$ & $\mathrm{C} 21-\mathrm{C} 22-\mathrm{N} 221-\mathrm{O} 221$ & $-17.4(6)$ \\
\hline $\mathrm{C} 111-\mathrm{N} 111-\mathrm{C} 112-\mathrm{N} 113$ & $179.8(5)$ & $\mathrm{C} 211-\mathrm{N} 211-\mathrm{C} 212-\mathrm{N} 213$ & $-170.5(4)$ \\
\hline $\mathrm{C} 111-\mathrm{N} 111-\mathrm{C} 112-\mathrm{S} 111$ & $-5.0(7)$ & $\mathrm{C} 211-\mathrm{N} 211-\mathrm{C} 212-\mathrm{S} 211$ & $10.9(6)$ \\
\hline $\mathrm{C} 17 \mathrm{~A}-\mathrm{S} 111-\mathrm{C} 112-\mathrm{N} 113$ & $1.6(4)$ & $\mathrm{C} 27 \mathrm{~A}-\mathrm{S} 211-\mathrm{C} 212-\mathrm{N} 213$ & $-1.8(4)$ \\
\hline $\mathrm{C} 17 \mathrm{~A}-\mathrm{S} 111-\mathrm{C} 112-\mathrm{N} 111$ & $-173.9(4)$ & $\mathrm{C} 27 \mathrm{~A}-\mathrm{S} 211-\mathrm{C} 212-\mathrm{N} 211$ & $176.9(4)$ \\
\hline $\mathrm{N} 111-\mathrm{C} 112-\mathrm{N} 113-\mathrm{C} 13 \mathrm{~A}$ & $173.7(4)$ & $\mathrm{N} 211-\mathrm{C} 212-\mathrm{N} 213-\mathrm{C} 23 \mathrm{~A}$ & $-179.2(4)$ \\
\hline $\mathrm{S} 111-\mathrm{C} 112-\mathrm{N} 113-\mathrm{C} 13 \mathrm{~A}$ & $-1.8(5)$ & $\mathrm{S} 211-\mathrm{C} 212-\mathrm{N} 213-\mathrm{C} 23 \mathrm{~A}$ & $-0.4(5)$ \\
\hline $\mathrm{C} 112-\mathrm{N} 113-\mathrm{C} 13 \mathrm{~A}-\mathrm{C} 17 \mathrm{~A}$ & $1.1(6)$ & $\mathrm{C} 212-\mathrm{N} 213-\mathrm{C} 23 \mathrm{~A}-\mathrm{C} 27 \mathrm{~A}$ & $3.3(5)$ \\
\hline $\mathrm{C} 112-\mathrm{N} 113-\mathrm{C} 13 \mathrm{~A}-\mathrm{C} 114$ & $-176.2(5)$ & $\mathrm{C} 212-\mathrm{N} 213-\mathrm{C} 23 \mathrm{~A}-\mathrm{C} 214$ & $-177.4(4)$ \\
\hline $\mathrm{C} 17 \mathrm{~A}-\mathrm{C} 13 \mathrm{~A}-\mathrm{C} 114-\mathrm{C} 115$ & $-0.5(7)$ & $\mathrm{N} 213-\mathrm{C} 23 \mathrm{~A}-\mathrm{C} 214-\mathrm{C} 215$ & $-177.2(4)$ \\
\hline $\mathrm{N} 113-\mathrm{C} 13 \mathrm{~A}-\mathrm{C} 114-\mathrm{C} 115$ & $176.7(4)$ & $\mathrm{C} 27 \mathrm{~A}-\mathrm{C} 23 \mathrm{~A}-\mathrm{C} 214-\mathrm{C} 215$ & $2.0(6)$ \\
\hline $\mathrm{C} 13 \mathrm{~A}-\mathrm{C} 114-\mathrm{C} 115-\mathrm{C} 116$ & $0.7(8)$ & $\mathrm{C} 23 \mathrm{~A}-\mathrm{C} 214-\mathrm{C} 215-\mathrm{C} 216$ & $2.8(7)$ \\
\hline $\mathrm{C} 114-\mathrm{C} 115-\mathrm{C} 116-\mathrm{O} 116$ & $179.1(4)$ & $\mathrm{C} 214-\mathrm{C} 215-\mathrm{C} 216-\mathrm{O} 216$ & $174.8(4)$ \\
\hline $\mathrm{C} 114-\mathrm{C} 115-\mathrm{C} 116-\mathrm{C} 117$ & $-0.1(8)$ & $\mathrm{C} 214-\mathrm{C} 215-\mathrm{C} 216-\mathrm{C} 217$ & $-6.3(7)$ \\
\hline $\mathrm{O} 116-\mathrm{C} 116-\mathrm{C} 117-\mathrm{C} 17 \mathrm{~A}$ & $-179.8(4)$ & $\mathrm{O} 216-\mathrm{C} 216-\mathrm{C} 217-\mathrm{C} 27 \mathrm{~A}$ & $-176.7(4)$ \\
\hline $\mathrm{C} 115-\mathrm{C} 116-\mathrm{C} 117-\mathrm{C} 17 \mathrm{~A}$ & $-0.7(7)$ & $\mathrm{C} 215-\mathrm{C} 216-\mathrm{C} 217-\mathrm{C} 27 \mathrm{~A}$ & $4.5(7)$ \\
\hline $\mathrm{C} 114-\mathrm{C} 13 \mathrm{~A}-\mathrm{C} 17 \mathrm{~A}-\mathrm{C} 117$ & $-0.3(7)$ & $\mathrm{C} 216-\mathrm{C} 217-\mathrm{C} 27 \mathrm{~A}-\mathrm{C} 23 \mathrm{~A}$ & $0.4(7)$ \\
\hline $\mathrm{N} 113-\mathrm{C} 13 \mathrm{~A}-\mathrm{C} 17 \mathrm{~A}-\mathrm{C} 117$ & $-177.9(4)$ & $\mathrm{C} 216-\mathrm{C} 217-\mathrm{C} 27 \mathrm{~A}-\mathrm{S} 211$ & $-179.4(4)$ \\
\hline $\mathrm{C} 114-\mathrm{C} 13 \mathrm{~A}-\mathrm{C} 17 \mathrm{~A}-\mathrm{S} 111$ & $177.6(4)$ & $\mathrm{N} 213-\mathrm{C} 23 \mathrm{~A}-\mathrm{C} 27 \mathrm{~A}-\mathrm{C} 217$ & $175.6(4)$ \\
\hline $\mathrm{N} 113-\mathrm{C} 13 \mathrm{~A}-\mathrm{C} 17 \mathrm{~A}-\mathrm{S} 111$ & $0.1(5)$ & $\mathrm{C} 214-\mathrm{C} 23 \mathrm{~A}-\mathrm{C} 27 \mathrm{~A}-\mathrm{C} 217$ & $-3.7(7)$ \\
\hline $\mathrm{C} 116-\mathrm{C} 117-\mathrm{C} 17 \mathrm{~A}-\mathrm{C} 13 \mathrm{~A}$ & $0.9(7)$ & $\mathrm{N} 213-\mathrm{C} 23 \mathrm{~A}-\mathrm{C} 27 \mathrm{~A}-\mathrm{S} 211$ & $-4.5(5)$ \\
\hline $\mathrm{C} 116-\mathrm{C} 117-\mathrm{C} 17 \mathrm{~A}-\mathrm{S} 111$ & $-176.6(4)$ & $\mathrm{C} 214-\mathrm{C} 23 \mathrm{~A}-\mathrm{C} 27 \mathrm{~A}-\mathrm{S} 211$ & $176.2(3)$ \\
\hline $\mathrm{C} 112-\mathrm{S} 111-\mathrm{C} 17 \mathrm{~A}-\mathrm{C} 13 \mathrm{~A}$ & $-0.8(3)$ & $\mathrm{C} 212-\mathrm{S} 211-\mathrm{C} 27 \mathrm{~A}-\mathrm{C} 217$ & $-176.8(4)$ \\
\hline
\end{tabular}




$\begin{array}{llll}\mathrm{C} 112-\mathrm{S} 111-\mathrm{C} 17 \mathrm{~A}-\mathrm{C} 117 & 177.0(5) & \mathrm{C} 212-\mathrm{S} 211-\mathrm{C} 27 \mathrm{~A}-\mathrm{C} 23 \mathrm{~A} & 3.4(4) \\ \mathrm{C} 117-\mathrm{C} 116-\mathrm{O} 116-\mathrm{C} 118 & 3.3(7) & \mathrm{C} 217-\mathrm{C} 216-\mathrm{O} 216-\mathrm{C} 218 & -0.3(7) \\ \mathrm{C} 115-\mathrm{C} 116-\mathrm{O} 116-\mathrm{C} 118 & -175.8(4) & \mathrm{C} 215-\mathrm{C} 216-\mathrm{O} 216-\mathrm{C} 218 & 178.6(4)\end{array}$

Hydrogen-bond geometry $\left(\AA,{ }^{\circ}\right)$

\begin{tabular}{lllll}
\hline$D-\mathrm{H} \cdots A$ & $D-\mathrm{H}$ & $\mathrm{H} \cdots A$ & $D \cdots A$ & $D-\mathrm{H} \cdots A$ \\
\hline $\mathrm{N} 111-\mathrm{H} 111 \cdots \mathrm{N} 213$ & $0.82(4)$ & $2.19(4)$ & $2.981(5)$ & $165(4)$ \\
$\mathrm{N} 211-\mathrm{H} 211 \cdots \mathrm{N} 113$ & $0.86(4)$ & $2.17(4)$ & $2.992(5)$ & $162(4)$ \\
$\mathrm{C} 13-\mathrm{H} 13 \cdots \mathrm{O} 211^{\mathrm{i}}$ & 0.93 & 2.53 & $3.408(7)$ & 158 \\
$\mathrm{C} 25-\mathrm{H} 25 \cdots \mathrm{O} 211^{\mathrm{ii}}$ & 0.93 & 2.44 & $3.349(6)$ & 165 \\
$\mathrm{C} 115-\mathrm{H} 115 \cdots \mathrm{O} 221^{\mathrm{ii}}$ & 0.93 & 2.45 & $3.353(7)$ & 163 \\
$\mathrm{C} 117-\mathrm{H} 117 \cdots \mathrm{O} 111^{\mathrm{iii}}$ & 0.93 & 2.44 & $3.236(5)$ & 144 \\
$\mathrm{C} 217-\mathrm{H} 217 \cdots \mathrm{O} 122^{\mathrm{iv}}$ & 0.93 & 2.51 & $3.412(6)$ & 164 \\
$\mathrm{C} 16-\mathrm{H} 16 \cdots C g 1^{\mathrm{v}}$ & 0.93 & 2.84 & $3.484(6)$ & 128 \\
\hline
\end{tabular}

Symmetry codes: (i) $-x+3 / 2, y+1 / 2, z-1 / 2$; (ii) $x, y, z+1$; (iii) $-x+1,-y+1, z+1 / 2$; (iv) $-x+3 / 2, y-1 / 2, z-1 / 2$; (v) $x, y, z-1$.

5-Cyclopropyl-N-(6-methoxybenzo[d] thiazol-2-yl)isoxazole-3-carboxamide (III)

Crystal data

$\mathrm{C}_{15} \mathrm{H}_{13} \mathrm{~N}_{3} \mathrm{O}_{3} \mathrm{~S}$

$M_{r}=315.34$

Monoclinic, $C 2 / c$

$a=18.720(1) \AA$

$b=11.5255(8) \AA$

$c=14.7905(9) \AA$

$\beta=115.52(1)^{\circ}$

$V=2879.8(4) \AA^{3}$

$Z=8$

\section{Data collection}

Oxford Diffraction Xcalibur CCD diffractometer

Radiation source: Enhance (Mo) X-ray Source Graphite monochromator

$\omega$ scans

Absorption correction: multi-scan

(CrysalisRed; Oxford Diffraction, 2009)

$T_{\min }=0.908, T_{\max }=0.976$

\section{Refinement}

Refinement on $F^{2}$

Least-squares matrix: full

$R\left[F^{2}>2 \sigma\left(F^{2}\right)\right]=0.056$

$w R\left(F^{2}\right)=0.119$

$S=1.02$

3118 reflections

268 parameters

26 restraints
$F(000)=1312$

$D_{\mathrm{x}}=1.455 \mathrm{Mg} \mathrm{m}^{-3}$

Mo $K \alpha$ radiation, $\lambda=0.71073 \AA$

Cell parameters from 3118 reflections

$\theta=2.9-27.8^{\circ}$

$\mu=0.24 \mathrm{~mm}^{-1}$

$T=296 \mathrm{~K}$

Block, colourless

$0.30 \times 0.20 \times 0.10 \mathrm{~mm}$

5937 measured reflections

3118 independent reflections

1730 reflections with $I>2 \sigma(I)$

$R_{\text {int }}=0.038$

$\theta_{\max }=27.8^{\circ}, \theta_{\min }=2.9^{\circ}$

$h=-24 \rightarrow 19$

$k=-15 \rightarrow 9$

$l=-18 \rightarrow 19$

Primary atom site location: difference Fourier map

Hydrogen site location: mixed

$\mathrm{H}$ atoms treated by a mixture of independent and constrained refinement

$w=1 /\left[\sigma^{2}\left(F_{\mathrm{o}}^{2}\right)+(0.0504 P)^{2}\right]$

where $P=\left(F_{\mathrm{o}}^{2}+2 F_{\mathrm{c}}^{2}\right) / 3$

$(\Delta / \sigma)_{\max }=0.001$

$\Delta \rho_{\max }=0.23 \mathrm{e} \AA^{-3}$

$\Delta \rho_{\min }=-0.24$ e $\AA^{-3}$ 


\section{Special details}

Geometry. All esds (except the esd in the dihedral angle between two 1.s. planes) are estimated using the full covariance matrix. The cell esds are taken into account individually in the estimation of esds in distances, angles and torsion angles; correlations between esds in cell parameters are only used when they are defined by crystal symmetry. An approximate (isotropic) treatment of cell esds is used for estimating esds involving l.s. planes.

Fractional atomic coordinates and isotropic or equivalent isotropic displacement parameters $\left(\AA^{2}\right)$

\begin{tabular}{|c|c|c|c|c|c|}
\hline & $x$ & $y$ & $z$ & $U_{\text {iso }} * / U_{\text {eq }}$ & Occ. $(<1)$ \\
\hline C31B & $0.45159(15)$ & $0.6697(2)$ & $0.4342(2)$ & $0.0494(7)$ & $0.451(5)$ \\
\hline O31B & $0.4916(12)$ & $0.682(3)$ & $0.5246(5)$ & $0.056(3)$ & $0.451(5)$ \\
\hline $\mathrm{O} 1 \mathrm{~B}$ & $0.2557(4)$ & $0.7800(10)$ & $0.2806(7)$ & $0.0511(13)$ & $0.451(5)$ \\
\hline $\mathrm{N} 2 \mathrm{~B}$ & $0.3373(6)$ & $0.766(3)$ & $0.3053(16)$ & $0.0495(13)$ & $0.451(5)$ \\
\hline $\mathrm{C} 3 \mathrm{~B}$ & $0.3650(8)$ & $0.703(4)$ & $0.387(3)$ & $0.0441(12)$ & $0.451(5)$ \\
\hline $\mathrm{C} 4 \mathrm{~B}$ & $0.3059(8)$ & $0.673(4)$ & $0.417(2)$ & $0.0492(13)$ & $0.451(5)$ \\
\hline H4B & 0.3114 & 0.6272 & 0.4713 & $0.059 *$ & $0.451(5)$ \\
\hline $\mathrm{C} 5 \mathrm{~B}$ & $0.2392(4)$ & $0.7242(13)$ & $0.3499(8)$ & $0.0446(17)$ & $0.451(5)$ \\
\hline C51 & $0.1558(4)$ & $0.7226(8)$ & $0.3338(5)$ & $0.057(2)$ & $0.451(5)$ \\
\hline H51 & 0.1464 & 0.6824 & 0.3860 & $0.068 *$ & $0.451(5)$ \\
\hline C52 & $0.0910(5)$ & $0.7088(8)$ & $0.2300(6)$ & $0.055(3)$ & $0.451(5)$ \\
\hline H52A & 0.1058 & 0.7077 & 0.1747 & $0.066^{*}$ & $0.451(5)$ \\
\hline H52B & 0.0467 & 0.6593 & 0.2214 & $0.066^{*}$ & $0.451(5)$ \\
\hline C53 & 0.0996 & $0.8175(6)$ & $0.2835(5)$ & $0.067(2)$ & $0.451(5)$ \\
\hline H53A & 0.0602 & 0.8357 & 0.3077 & $0.080^{*}$ & $0.451(5)$ \\
\hline H53B & 0.1194 & 0.8841 & 0.2611 & $0.080^{*}$ & $0.451(5)$ \\
\hline C31A & $0.45159(15)$ & $0.6697(2)$ & $0.4342(2)$ & $0.0494(7)$ & $0.549(5)$ \\
\hline O31A & $0.4786(9)$ & $0.655(2)$ & $0.5251(4)$ & $0.056(3)$ & $0.549(5)$ \\
\hline O1A & $0.2719(3)$ & $0.8029(8)$ & $0.2617(5)$ & $0.0511(13)$ & $0.549(5)$ \\
\hline $\mathrm{N} 2 \mathrm{~A}$ & $0.3533(5)$ & $0.778(2)$ & $0.2975(13)$ & $0.0495(13)$ & $0.549(5)$ \\
\hline $\mathrm{C} 3 \mathrm{~A}$ & 0.3683 (6) & $0.710(4)$ & $0.374(2)$ & $0.0441(12)$ & $0.549(5)$ \\
\hline $\mathrm{C} 4 \mathrm{~A}$ & $0.3025(6)$ & $0.690(3)$ & $0.3936(17)$ & $0.0492(13)$ & $0.549(5)$ \\
\hline $\mathrm{H} 4 \mathrm{~A}$ & 0.3002 & 0.6471 & 0.4455 & $0.059 *$ & $0.549(5)$ \\
\hline $\mathrm{C} 5 \mathrm{~A}$ & 0.2435 & $0.7473(10)$ & $0.3203(7)$ & $0.0446(17)$ & $0.549(5)$ \\
\hline C61 & $0.1581(3)$ & $0.7625(6)$ & $0.2904(5)$ & $0.058(2)$ & $0.549(5)$ \\
\hline H61 & 0.1352 & 0.8351 & 0.2554 & $0.069 *$ & $0.549(5)$ \\
\hline C62 & $0.1039(5)$ & $0.6592(7)$ & $0.2597(6)$ & $0.067(3)$ & $0.549(5)$ \\
\hline H62A & 0.0511 & 0.6696 & 0.2064 & $0.080 *$ & $0.549(5)$ \\
\hline H62B & 0.1272 & 0.5836 & 0.2613 & $0.080 *$ & $0.549(5)$ \\
\hline C63 & $0.1224(3)$ & $0.7162(6)$ & $0.3547(4)$ & $0.068(2)$ & $0.549(5)$ \\
\hline H63A & 0.1569 & 0.6757 & 0.4153 & $0.082 *$ & $0.549(5)$ \\
\hline H63B & 0.0809 & 0.7616 & 0.3605 & $0.082 *$ & $0.549(5)$ \\
\hline N31 & $0.48732(12)$ & $0.6444(2)$ & $0.37429(16)$ & $0.0474(6)$ & \\
\hline H31 & $0.4615(16)$ & $0.637(2)$ & $0.314(2)$ & $0.057^{*}$ & \\
\hline S11 & $0.63319(4)$ & $0.63305(6)$ & $0.53535(5)$ & $0.0480(2)$ & \\
\hline $\mathrm{C} 12$ & $0.56815(13)$ & $0.6228(2)$ & $0.40899(18)$ & 0.0404 (6) & \\
\hline N13 & $0.59782(12)$ & 0.59878 (19) & $0.34675(15)$ & $0.0460(6)$ & \\
\hline C13A & $0.68009(15)$ & $0.5844(2)$ & $0.39970(19)$ & $0.0436(6)$ & \\
\hline $\mathrm{C} 14$ & $0.73158(16)$ & $0.5543(2)$ & $0.3582(2)$ & $0.0556(8)$ & \\
\hline
\end{tabular}




\begin{tabular}{|c|c|c|c|c|c|}
\hline H14 & 0.7124 & 0.5405 & 0.2898 & $0.067^{*}$ & \\
\hline $\mathrm{C} 15$ & $0.81099(17)$ & $0.5452(3)$ & $0.4194(2)$ & $0.0581(8)$ & \\
\hline H15 & 0.8454 & 0.5232 & 0.3921 & $0.070^{*}$ & \\
\hline $\mathrm{C} 17$ & $0.79097(14)$ & $0.5970(2)$ & $0.5647(2)$ & $0.0490(7)$ & \\
\hline H17 & 0.8106 & 0.6113 & 0.6330 & $0.059 *$ & \\
\hline C17A & $0.71007(14)$ & $0.6035(2)$ & $0.50220(19)$ & $0.0406(6)$ & \\
\hline $\mathrm{C} 16 \mathrm{~A}$ & $0.84091(15)$ & $0.5684(2)$ & $0.5213(2)$ & $0.0528(7)$ & 0.5 \\
\hline O16 & $0.9220(6)$ & $0.552(5)$ & $0.577(3)$ & $0.065(7)$ & 0.5 \\
\hline $\mathrm{C} 18$ & $0.9590(3)$ & $0.5892(5)$ & $0.6867(4)$ & $0.0638(16)$ & 0.5 \\
\hline $\mathrm{H} 18 \mathrm{~A}$ & 1.0156 & 0.5873 & 0.7124 & $0.096^{*}$ & 0.5 \\
\hline H18B & 0.9424 & 0.5369 & 0.7246 & $0.096^{*}$ & 0.5 \\
\hline $\mathrm{H} 18 \mathrm{C}$ & 0.9422 & 0.6665 & 0.6922 & $0.096^{*}$ & 0.5 \\
\hline $\mathrm{C} 16 \mathrm{~B}$ & $0.84091(15)$ & $0.5684(2)$ & $0.5213(2)$ & $0.0528(7)$ & 0.5 \\
\hline $\mathrm{O} 17$ & $0.9206(5)$ & $0.576(5)$ & $0.581(3)$ & $0.061(6)$ & 0.5 \\
\hline C19 & $0.9693(3)$ & $0.5307(6)$ & $0.5324(4)$ & $0.0719(19)$ & 0.5 \\
\hline H19A & 1.0243 & 0.5366 & 0.5780 & $0.108^{*}$ & 0.5 \\
\hline H19B & 0.9591 & 0.5753 & 0.4733 & $0.108^{*}$ & 0.5 \\
\hline H19C & 0.9561 & 0.4509 & 0.5143 & $0.108^{*}$ & 0.5 \\
\hline
\end{tabular}

Atomic displacement parameters $\left(\AA^{2}\right)$

\begin{tabular}{lllllll}
\hline & $U^{11}$ & $U^{22}$ & $U^{33}$ & $U^{12}$ & $U^{13}$ & $U^{23}$ \\
\hline C31B & $0.0317(14)$ & $0.065(2)$ & $0.0495(16)$ & $-0.0006(13)$ & $0.0160(13)$ & $0.0014(15)$ \\
O31B & $0.024(5)$ & $0.096(10)$ & $0.0478(12)$ & $-0.009(3)$ & $0.0161(11)$ & $0.0036(15)$ \\
O1B & $0.030(3)$ & $0.072(4)$ & $0.055(3)$ & $0.008(2)$ & $0.0215(15)$ & $0.0136(19)$ \\
N2B & $0.023(3)$ & $0.068(5)$ & $0.059(3)$ & $0.002(5)$ & $0.019(3)$ & $0.0044(17)$ \\
C3B & $0.0288(15)$ & $0.053(4)$ & $0.049(6)$ & $-0.001(2)$ & $0.0146(14)$ & $0.002(3)$ \\
C4B & $0.0340(18)$ & $0.053(8)$ & $0.062(9)$ & $0.0033(16)$ & $0.022(3)$ & $0.017(4)$ \\
C5B & $0.0375(19)$ & $0.055(5)$ & $0.051(5)$ & $-0.002(2)$ & $0.028(2)$ & $0.004(4)$ \\
C51 & $0.039(4)$ & $0.084(7)$ & $0.058(5)$ & $0.002(5)$ & $0.032(4)$ & $0.011(5)$ \\
C52 & $0.033(4)$ & $0.058(7)$ & $0.077(6)$ & $-0.007(4)$ & $0.026(4)$ & $0.005(5)$ \\
C53 & $0.043(4)$ & $0.058(5)$ & $0.103(6)$ & $-0.002(3)$ & $0.035(4)$ & $-0.007(5)$ \\
C31A & $0.0317(14)$ & $0.065(2)$ & $0.0495(16)$ & $-0.0006(13)$ & $0.0160(13)$ & $0.0014(15)$ \\
O31A & $0.024(5)$ & $0.096(10)$ & $0.0478(12)$ & $-0.009(3)$ & $0.0161(11)$ & $0.0036(15)$ \\
O1A & $0.030(3)$ & $0.072(4)$ & $0.055(3)$ & $0.008(2)$ & $0.0215(15)$ & $0.0136(19)$ \\
N2A & $0.023(3)$ & $0.068(5)$ & $0.059(3)$ & $0.002(5)$ & $0.019(3)$ & $0.0044(17)$ \\
C3A & $0.0288(15)$ & $0.053(4)$ & $0.049(6)$ & $-0.001(2)$ & $0.0146(14)$ & $0.002(3)$ \\
C4A & $0.0340(18)$ & $0.053(8)$ & $0.062(9)$ & $0.0033(16)$ & $0.022(3)$ & $0.017(4)$ \\
C5A & $0.0375(19)$ & $0.055(5)$ & $0.051(5)$ & $-0.002(2)$ & $0.028(2)$ & $0.004(4)$ \\
C61 & $0.028(3)$ & $0.074(5)$ & $0.074(5)$ & $0.007(3)$ & $0.024(3)$ & $0.013(4)$ \\
C62 & $0.035(4)$ & $0.087(7)$ & $0.079(5)$ & $-0.011(5)$ & $0.025(4)$ & $-0.018(6)$ \\
C63 & $0.039(4)$ & $0.109(6)$ & $0.067(4)$ & $0.001(4)$ & $0.033(3)$ & $0.000(4)$ \\
N31 & $0.0273(11)$ & $0.0672(16)$ & $0.0421(12)$ & $0.0001(11)$ & $0.0096(10)$ & $-0.0008(13)$ \\
S11 & $0.0288(3)$ & $0.0700(5)$ & $0.0427(4)$ & $0.0041(3)$ & $0.0131(3)$ & $-0.0019(4)$ \\
C12 & $0.0298(13)$ & $0.0478(17)$ & $0.0410(13)$ & $0.0009(12)$ & $0.0127(11)$ & $-0.0001(13)$ \\
N13 & $0.0367(12)$ & $0.0544(15)$ & $0.0441(12)$ & $0.0048(10)$ & $0.0146(10)$ & $-0.0003(11)$ \\
C13A & $0.0395(15)$ & $0.0444(17)$ & $0.0486(16)$ & $0.0060(13)$ & $0.0204(13)$ & $0.0046(13)$ \\
C14 & $0.0545(18)$ & $0.064(2)$ & $0.0533(17)$ & $0.0134(15)$ & $0.0280(15)$ & $0.0053(15)$ \\
& & & & &
\end{tabular}


supporting information

\begin{tabular}{lllllll} 
C15 & $0.0493(18)$ & $0.067(2)$ & $0.071(2)$ & $0.0172(15)$ & $0.0378(16)$ & $0.0107(17)$ \\
C17 & $0.0335(14)$ & $0.0581(19)$ & $0.0534(16)$ & $0.0036(13)$ & $0.0168(13)$ & $-0.0011(14)$ \\
C17A & $0.0321(13)$ & $0.0413(16)$ & $0.0490(15)$ & $0.0024(11)$ & $0.0180(12)$ & $0.0013(12)$ \\
C16A & $0.0331(15)$ & $0.0533(19)$ & $0.073(2)$ & $0.0085(13)$ & $0.0232(15)$ & $0.0127(16)$ \\
O16 & $0.038(6)$ & $0.081(17)$ & $0.067(8)$ & $0.012(4)$ & $0.014(5)$ & $0.001(8)$ \\
C18 & $0.039(3)$ & $0.064(4)$ & $0.070(4)$ & $-0.001(3)$ & $0.006(3)$ & $0.011(3)$ \\
C16B & $0.0331(15)$ & $0.0533(19)$ & $0.073(2)$ & $0.0085(13)$ & $0.0232(15)$ & $0.0127(16)$ \\
O17 & $0.031(5)$ & $0.084(18)$ & $0.069(7)$ & $0.013(4)$ & $0.022(5)$ & $-0.008(7)$ \\
C19 & $0.032(3)$ & $0.113(6)$ & $0.074(4)$ & $0.009(3)$ & $0.026(3)$ & $0.003(4)$ \\
\hline
\end{tabular}

Geometric parameters $\left(\AA,{ }^{\circ}\right)$

\begin{tabular}{|c|c|c|c|}
\hline $\mathrm{C} 31 \mathrm{~B}-\mathrm{O} 31 \mathrm{~B}$ & $1.227(7)$ & $\mathrm{C} 62-\mathrm{H} 62 \mathrm{~B}$ & 0.9700 \\
\hline $\mathrm{C} 31 \mathrm{~B}-\mathrm{N} 31$ & $1.352(3)$ & $\mathrm{C} 63-\mathrm{H} 63 \mathrm{~A}$ & 0.9700 \\
\hline $\mathrm{C} 31 \mathrm{~B}-\mathrm{C} 3 \mathrm{~B}$ & $1.512(6)$ & $\mathrm{C} 63-\mathrm{H} 63 \mathrm{~B}$ & 0.9700 \\
\hline $\mathrm{O} 1 \mathrm{~B}-\mathrm{C} 5 \mathrm{~B}$ & $1.353(6)$ & $\mathrm{N} 31-\mathrm{C} 12$ & $1.395(3)$ \\
\hline $\mathrm{O} 1 \mathrm{~B}-\mathrm{N} 2 \mathrm{~B}$ & $1.419(6)$ & $\mathrm{N} 31-\mathrm{H} 31$ & $0.82(3)$ \\
\hline $\mathrm{N} 2 \mathrm{~B}-\mathrm{C} 3 \mathrm{~B}$ & $1.310(6)$ & $\mathrm{S} 11-\mathrm{C} 12$ & $1.740(2)$ \\
\hline $\mathrm{C} 3 \mathrm{~B}-\mathrm{C} 4 \mathrm{~B}$ & $1.401(6)$ & $\mathrm{S} 11-\mathrm{C} 17 \mathrm{~A}$ & $1.742(2)$ \\
\hline $\mathrm{C} 4 \mathrm{~B}-\mathrm{C} 5 \mathrm{~B}$ & $1.350(7)$ & $\mathrm{C} 12-\mathrm{N} 13$ & $1.293(3)$ \\
\hline $\mathrm{C} 4 \mathrm{~B}-\mathrm{H} 4 \mathrm{~B}$ & 0.9300 & $\mathrm{~N} 13-\mathrm{C} 13 \mathrm{~A}$ & $1.405(3)$ \\
\hline $\mathrm{C} 5 \mathrm{~B}-\mathrm{C} 51$ & $1.474(7)$ & $\mathrm{C} 13 \mathrm{~A}-\mathrm{C} 17 \mathrm{~A}$ & $1.389(3)$ \\
\hline $\mathrm{C} 51-\mathrm{C} 53$ & $1.477(7)$ & $\mathrm{C} 13 \mathrm{~A}-\mathrm{C} 14$ & $1.391(3)$ \\
\hline $\mathrm{C} 51-\mathrm{C} 52$ & $1.499(7)$ & $\mathrm{C} 14-\mathrm{C} 15$ & $1.371(4)$ \\
\hline C51-H51 & 0.9800 & $\mathrm{C} 14-\mathrm{H} 14$ & 0.9300 \\
\hline $\mathrm{C} 52-\mathrm{C} 53$ & $1.454(8)$ & $\mathrm{C} 15-\mathrm{C} 16 \mathrm{~A}$ & $1.389(4)$ \\
\hline $\mathrm{C} 52-\mathrm{H} 52 \mathrm{~A}$ & 0.9700 & $\mathrm{C} 15-\mathrm{H} 15$ & 0.9300 \\
\hline C52-H52B & 0.9700 & $\mathrm{C} 17-\mathrm{C} 16 \mathrm{~A}$ & $1.383(3)$ \\
\hline C53-H53A & 0.9700 & $\mathrm{C} 17-\mathrm{C} 17 \mathrm{~A}$ & $1.395(3)$ \\
\hline C53-H53B & 0.9700 & $\mathrm{C} 17-\mathrm{H} 17$ & 0.9300 \\
\hline $\mathrm{O} 1 \mathrm{~A}-\mathrm{C} 5 \mathrm{~A}$ & $1.357(5)$ & $\mathrm{C} 16 \mathrm{~A}-\mathrm{O} 16$ & $1.393(8)$ \\
\hline $\mathrm{O} 1 \mathrm{~A}-\mathrm{N} 2 \mathrm{~A}$ & $1.412(5)$ & $\mathrm{O} 16-\mathrm{C} 18$ & $1.52(4)$ \\
\hline $\mathrm{N} 2 \mathrm{~A}-\mathrm{C} 3 \mathrm{~A}$ & $1.308(6)$ & $\mathrm{C} 18-\mathrm{C} 18^{\mathrm{i}}$ & $1.840(10)$ \\
\hline $\mathrm{C} 3 \mathrm{~A}-\mathrm{C} 4 \mathrm{~A}$ & $1.399(5)$ & $\mathrm{C} 18-\mathrm{H} 18 \mathrm{~A}$ & 0.9600 \\
\hline $\mathrm{C} 4 \mathrm{~A}-\mathrm{C} 5 \mathrm{~A}$ & $1.342(6)$ & $\mathrm{C} 18-\mathrm{H} 18 \mathrm{~B}$ & 0.9600 \\
\hline $\mathrm{C} 4 \mathrm{~A}-\mathrm{H} 4 \mathrm{~A}$ & 0.9300 & $\mathrm{C} 18-\mathrm{H} 18 \mathrm{C}$ & 0.9600 \\
\hline $\mathrm{C} 5 \mathrm{~A}-\mathrm{C} 61$ & $1.473(6)$ & $\mathrm{O} 17-\mathrm{C} 19$ & $1.48(4)$ \\
\hline $\mathrm{C} 61-\mathrm{C} 63$ & $1.478(6)$ & $\mathrm{C} 19-\mathrm{C} 19^{\mathrm{ii}}$ & $1.920(11)$ \\
\hline $\mathrm{C} 61-\mathrm{C} 62$ & $1.503(7)$ & C19-H19A & 0.9600 \\
\hline C61-H61 & 0.9800 & C19-H19B & 0.9600 \\
\hline $\mathrm{C} 62-\mathrm{C} 63$ & $1.451(7)$ & $\mathrm{C} 19-\mathrm{H} 19 \mathrm{C}$ & 0.9600 \\
\hline $\mathrm{C} 62-\mathrm{H} 62 \mathrm{~A}$ & 0.9700 & & \\
\hline $\mathrm{O} 31 \mathrm{~B}-\mathrm{C} 31 \mathrm{~B}-\mathrm{N} 31$ & $119.8(12)$ & $\mathrm{C} 62-\mathrm{C} 63-\mathrm{C} 61$ & $61.8(4)$ \\
\hline $\mathrm{O} 31 \mathrm{~B}-\mathrm{C} 31 \mathrm{~B}-\mathrm{C} 3 \mathrm{~B}$ & $120.3(12)$ & $\mathrm{C} 62-\mathrm{C} 63-\mathrm{H} 63 \mathrm{~A}$ & 117.6 \\
\hline $\mathrm{N} 31-\mathrm{C} 31 \mathrm{~B}-\mathrm{C} 3 \mathrm{~B}$ & $119.0(14)$ & $\mathrm{C} 61-\mathrm{C} 63-\mathrm{H} 63 \mathrm{~A}$ & 117.6 \\
\hline $\mathrm{C} 5 \mathrm{~B}-\mathrm{O} 1 \mathrm{~B}-\mathrm{N} 2 \mathrm{~B}$ & $108.9(5)$ & $\mathrm{C} 62-\mathrm{C} 63-\mathrm{H} 63 \mathrm{~B}$ & 117.6 \\
\hline $\mathrm{C} 3 \mathrm{~B}-\mathrm{N} 2 \mathrm{~B}-\mathrm{O} 1 \mathrm{~B}$ & $104.8(5)$ & C61-C63-H63B & 117.6 \\
\hline
\end{tabular}




\begin{tabular}{|c|c|}
\hline $\mathrm{N} 2 \mathrm{~B}-\mathrm{C} 3 \mathrm{~B}-\mathrm{C} 4 \mathrm{~B}$ & $112.0(5)$ \\
\hline $\mathrm{N} 2 \mathrm{~B}-\mathrm{C} 3 \mathrm{~B}-\mathrm{C} 31 \mathrm{~B}$ & $119.2(7)$ \\
\hline $\mathrm{C} 4 \mathrm{~B}-\mathrm{C} 3 \mathrm{~B}-\mathrm{C} 31 \mathrm{~B}$ & $128.8(7)$ \\
\hline $\mathrm{C} 5 \mathrm{~B}-\mathrm{C} 4 \mathrm{~B}-\mathrm{C} 3 \mathrm{~B}$ & $105.5(6)$ \\
\hline $\mathrm{C} 5 \mathrm{~B}-\mathrm{C} 4 \mathrm{~B}-\mathrm{H} 4 \mathrm{~B}$ & 127.3 \\
\hline $\mathrm{C} 3 \mathrm{~B}-\mathrm{C} 4 \mathrm{~B}-\mathrm{H} 4 \mathrm{~B}$ & 127.3 \\
\hline $\mathrm{C} 4 \mathrm{~B}-\mathrm{C} 5 \mathrm{~B}-\mathrm{O} 1 \mathrm{~B}$ & $108.8(6)$ \\
\hline $\mathrm{C} 4 \mathrm{~B}-\mathrm{C} 5 \mathrm{~B}-\mathrm{C} 51$ & $133.9(7)$ \\
\hline $\mathrm{O} 1 \mathrm{~B}-\mathrm{C} 5 \mathrm{~B}-\mathrm{C} 51$ & $117.1(6)$ \\
\hline $\mathrm{C} 5 \mathrm{~B}-\mathrm{C} 51-\mathrm{C} 53$ & $123.2(8)$ \\
\hline $\mathrm{C} 5 \mathrm{~B}-\mathrm{C} 51-\mathrm{C} 52$ & $120.0(7)$ \\
\hline C53-C51-C52 & $58.5(4)$ \\
\hline $\mathrm{C} 5 \mathrm{~B}-\mathrm{C} 51-\mathrm{H} 51$ & 114.6 \\
\hline C53-C51-H51 & 114.6 \\
\hline $\mathrm{C} 52-\mathrm{C} 51-\mathrm{H} 51$ & 114.6 \\
\hline $\mathrm{C} 53-\mathrm{C} 52-\mathrm{C} 51$ & $60.0(4)$ \\
\hline $\mathrm{C} 53-\mathrm{C} 52-\mathrm{H} 52 \mathrm{~A}$ & 117.8 \\
\hline $\mathrm{C} 51-\mathrm{C} 52-\mathrm{H} 52 \mathrm{~A}$ & 117.8 \\
\hline $\mathrm{C} 53-\mathrm{C} 52-\mathrm{H} 52 \mathrm{~B}$ & 117.8 \\
\hline С $51-\mathrm{C} 52-\mathrm{H} 52 \mathrm{~B}$ & 117.8 \\
\hline $\mathrm{H} 52 \mathrm{~A}-\mathrm{C} 52-\mathrm{H} 52 \mathrm{~B}$ & 114.9 \\
\hline $\mathrm{C} 52-\mathrm{C} 53-\mathrm{C} 51$ & $61.5(4)$ \\
\hline $\mathrm{C} 52-\mathrm{C} 53-\mathrm{H} 53 \mathrm{~A}$ & 117.6 \\
\hline $\mathrm{C} 51-\mathrm{C} 53-\mathrm{H} 53 \mathrm{~A}$ & 117.6 \\
\hline С $52-\mathrm{C} 53-\mathrm{H} 53 \mathrm{~B}$ & 117.6 \\
\hline $\mathrm{C} 51-\mathrm{C} 53-\mathrm{H} 53 \mathrm{~B}$ & 117.6 \\
\hline $\mathrm{H} 53 \mathrm{~A}-\mathrm{C} 53-\mathrm{H} 53 \mathrm{~B}$ & 114.7 \\
\hline $\mathrm{C} 5 \mathrm{~A}-\mathrm{O} 1 \mathrm{~A}-\mathrm{N} 2 \mathrm{~A}$ & $108.8(4)$ \\
\hline $\mathrm{C} 3 \mathrm{~A}-\mathrm{N} 2 \mathrm{~A}-\mathrm{O} 1 \mathrm{~A}$ & $103.9(4)$ \\
\hline $\mathrm{N} 2 \mathrm{~A}-\mathrm{C} 3 \mathrm{~A}-\mathrm{C} 4 \mathrm{~A}$ & $113.4(5)$ \\
\hline $\mathrm{C} 5 \mathrm{~A}-\mathrm{C} 4 \mathrm{~A}-\mathrm{C} 3 \mathrm{~A}$ & $104.2(5)$ \\
\hline $\mathrm{C} 5 \mathrm{~A}-\mathrm{C} 4 \mathrm{~A}-\mathrm{H} 4 \mathrm{~A}$ & 127.9 \\
\hline $\mathrm{C} 3 \mathrm{~A}-\mathrm{C} 4 \mathrm{~A}-\mathrm{H} 4 \mathrm{~A}$ & 127.9 \\
\hline $\mathrm{C} 4 \mathrm{~A}-\mathrm{C} 5 \mathrm{~A}-\mathrm{O} 1 \mathrm{~A}$ & $109.6(5)$ \\
\hline $\mathrm{C} 4 \mathrm{~A}-\mathrm{C} 5 \mathrm{~A}-\mathrm{C} 61$ & $135.3(6)$ \\
\hline $\mathrm{O} 1 \mathrm{~A}-\mathrm{C} 5 \mathrm{~A}-\mathrm{C} 61$ & $115.1(5)$ \\
\hline $\mathrm{C} 5 \mathrm{~A}-\mathrm{C} 61-\mathrm{C} 63$ & $119.8(5)$ \\
\hline $\mathrm{C} 5 \mathrm{~A}-\mathrm{C} 61-\mathrm{C} 62$ & $120.0(7)$ \\
\hline $\mathrm{C} 63-\mathrm{C} 61-\mathrm{C} 62$ & $58.2(4)$ \\
\hline $\mathrm{C} 5 \mathrm{~A}-\mathrm{C} 61-\mathrm{H} 61$ & 115.6 \\
\hline $\mathrm{C} 63-\mathrm{C} 61-\mathrm{H} 61$ & 115.6 \\
\hline $\mathrm{C} 62-\mathrm{C} 61-\mathrm{H} 61$ & 115.6 \\
\hline $\mathrm{C} 63-\mathrm{C} 62-\mathrm{C} 61$ & $60.0(3)$ \\
\hline $\mathrm{C} 63-\mathrm{C} 62-\mathrm{H} 62 \mathrm{~A}$ & 117.8 \\
\hline $\mathrm{C} 61-\mathrm{C} 62-\mathrm{H} 62 \mathrm{~A}$ & 117.8 \\
\hline C63-C62-H62B & 117.8 \\
\hline C61-C62-H62B & 117.8 \\
\hline & \\
\hline
\end{tabular}

\begin{tabular}{|c|c|}
\hline $\mathrm{H} 63 \mathrm{~A}-\mathrm{C} 63-\mathrm{H} 63 \mathrm{~B}$ & 114.7 \\
\hline $\mathrm{C} 31 \mathrm{~B}-\mathrm{N} 31-\mathrm{C} 12$ & $124.2(2)$ \\
\hline $\mathrm{C} 31 \mathrm{~B}-\mathrm{N} 31-\mathrm{H} 31$ & $120.8(19)$ \\
\hline $\mathrm{C} 12-\mathrm{N} 31-\mathrm{H} 31$ & $114.9(19)$ \\
\hline $\mathrm{C} 12-\mathrm{S} 11-\mathrm{C} 17 \mathrm{~A}$ & $87.99(11)$ \\
\hline $\mathrm{N} 13-\mathrm{C} 12-\mathrm{N} 31$ & $120.4(2)$ \\
\hline $\mathrm{N} 13-\mathrm{C} 12-\mathrm{S} 11$ & $117.56(18)$ \\
\hline $\mathrm{N} 31-\mathrm{C} 12-\mathrm{S} 11$ & $121.94(18)$ \\
\hline $\mathrm{C} 12-\mathrm{N} 13-\mathrm{C} 13 \mathrm{~A}$ & $109.4(2)$ \\
\hline $\mathrm{C} 17 \mathrm{~A}-\mathrm{C} 13 \mathrm{~A}-\mathrm{C} 14$ & $119.4(2)$ \\
\hline $\mathrm{C} 17 \mathrm{~A}-\mathrm{C} 13 \mathrm{~A}-\mathrm{N} 13$ & $114.9(2)$ \\
\hline $\mathrm{C} 14-\mathrm{C} 13 \mathrm{~A}-\mathrm{N} 13$ & $125.8(2)$ \\
\hline $\mathrm{C} 15-\mathrm{C} 14-\mathrm{C} 13 \mathrm{~A}$ & $119.2(3)$ \\
\hline $\mathrm{C} 15-\mathrm{C} 14-\mathrm{H} 14$ & 120.4 \\
\hline $\mathrm{C} 13 \mathrm{~A}-\mathrm{C} 14-\mathrm{H} 14$ & 120.4 \\
\hline $\mathrm{C} 14-\mathrm{C} 15-\mathrm{C} 16 \mathrm{~A}$ & $121.1(3)$ \\
\hline $\mathrm{C} 14-\mathrm{C} 15-\mathrm{H} 15$ & 119.4 \\
\hline $\mathrm{C} 16 \mathrm{~A}-\mathrm{C} 15-\mathrm{H} 15$ & 119.4 \\
\hline $\mathrm{C} 16 \mathrm{~A}-\mathrm{C} 17-\mathrm{C} 17 \mathrm{~A}$ & $117.6(3)$ \\
\hline $\mathrm{C} 16 \mathrm{~A}-\mathrm{C} 17-\mathrm{H} 17$ & 121.2 \\
\hline $\mathrm{C} 17 \mathrm{~A}-\mathrm{C} 17-\mathrm{H} 17$ & 121.2 \\
\hline $\mathrm{C} 13 \mathrm{~A}-\mathrm{C} 17 \mathrm{~A}-\mathrm{C} 17$ & $121.8(2)$ \\
\hline $\mathrm{C} 13 \mathrm{~A}-\mathrm{C} 17 \mathrm{~A}-\mathrm{S} 11$ & $110.11(18)$ \\
\hline $\mathrm{C} 17-\mathrm{C} 17 \mathrm{~A}-\mathrm{S} 11$ & $128.1(2)$ \\
\hline $\mathrm{C} 17-\mathrm{C} 16 \mathrm{~A}-\mathrm{C} 15$ & $120.8(3)$ \\
\hline $\mathrm{C} 17-\mathrm{C} 16 \mathrm{~A}-\mathrm{O} 16$ & $123(2)$ \\
\hline $\mathrm{C} 15-\mathrm{C} 16 \mathrm{~A}-\mathrm{O} 16$ & $116(2)$ \\
\hline $\mathrm{C} 16 \mathrm{~A}-\mathrm{O} 16-\mathrm{C} 18$ & $118(3)$ \\
\hline $\mathrm{O} 16-\mathrm{C} 18-\mathrm{C} 18^{\mathrm{i}}$ & $151.6(14)$ \\
\hline $\mathrm{O} 16-\mathrm{C} 18-\mathrm{H} 18 \mathrm{~A}$ & 109.5 \\
\hline 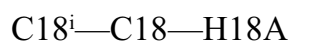 & 45.7 \\
\hline $\mathrm{O} 16-\mathrm{C} 18-\mathrm{H} 18 \mathrm{~B}$ & 109.5 \\
\hline $\mathrm{C} 18^{\mathrm{i}}-\mathrm{C} 18-\mathrm{H} 18 \mathrm{~B}$ & 75.2 \\
\hline $\mathrm{H} 18 \mathrm{~A}-\mathrm{C} 18-\mathrm{H} 18 \mathrm{~B}$ & 109.5 \\
\hline $\mathrm{O} 16-\mathrm{C} 18-\mathrm{H} 18 \mathrm{C}$ & 109.5 \\
\hline 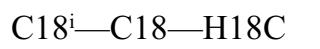 & 94.3 \\
\hline $\mathrm{H} 18 \mathrm{~A}-\mathrm{C} 18-\mathrm{H} 18 \mathrm{C}$ & 109.5 \\
\hline $\mathrm{H} 18 \mathrm{~B}-\mathrm{C} 18-\mathrm{H} 18 \mathrm{C}$ & 109.5 \\
\hline $\mathrm{O} 17-\mathrm{C} 19-\mathrm{C} 19^{\mathrm{ii}}$ & $178.6(18)$ \\
\hline $\mathrm{O} 17-\mathrm{C} 19-\mathrm{H} 19 \mathrm{~A}$ & 109.5 \\
\hline $\mathrm{C} 19^{\mathrm{ii}}-\mathrm{C} 19-\mathrm{H} 19 \mathrm{~A}$ & 71.7 \\
\hline $\mathrm{O} 17-\mathrm{C} 19-\mathrm{H} 19 \mathrm{~B}$ & 109.5 \\
\hline $\mathrm{C} 19^{\mathrm{ii}}-\mathrm{C} 19-\mathrm{H} 19 \mathrm{~B}$ & 70.7 \\
\hline $\mathrm{H} 19 \mathrm{~A}-\mathrm{C} 19-\mathrm{H} 19 \mathrm{~B}$ & 109.5 \\
\hline $\mathrm{O} 17-\mathrm{C} 19-\mathrm{H} 19 \mathrm{C}$ & 109.5 \\
\hline $\mathrm{C} 19^{\mathrm{ii}}-\mathrm{C} 19-\mathrm{H} 19 \mathrm{C}$ & 69.2 \\
\hline $\mathrm{H} 19 \mathrm{~A}-\mathrm{C} 19-\mathrm{H} 19 \mathrm{C}$ & 109.5 \\
\hline $\mathrm{H} 19 \mathrm{~B}-\mathrm{C} 19-\mathrm{H} 19 \mathrm{C}$ & 109.5 \\
\hline
\end{tabular}




\begin{tabular}{|c|c|c|c|}
\hline $\mathrm{C} 5 \mathrm{~B}-\mathrm{O} 1 \mathrm{~B}-\mathrm{N} 2 \mathrm{~B}-\mathrm{C} 3 \mathrm{~B}$ & $0(4)$ & $\mathrm{C} 5 \mathrm{~A}-\mathrm{C} 61-\mathrm{C} 62-\mathrm{C} 63$ & $108.5(7)$ \\
\hline $\mathrm{O} 1 \mathrm{~B}-\mathrm{N} 2 \mathrm{~B}-\mathrm{C} 3 \mathrm{~B}-\mathrm{C} 4 \mathrm{~B}$ & $1(6)$ & $\mathrm{C} 5 \mathrm{~A}-\mathrm{C} 61-\mathrm{C} 63-\mathrm{C} 62$ & $-108.9(8)$ \\
\hline $\mathrm{O} 1 \mathrm{~B}-\mathrm{N} 2 \mathrm{~B}-\mathrm{C} 3 \mathrm{~B}-\mathrm{C} 31 \mathrm{~B}$ & $179(4)$ & $\mathrm{O} 31 \mathrm{~B}-\mathrm{C} 31 \mathrm{~B}-\mathrm{N} 31-\mathrm{C} 12$ & $4.6(15)$ \\
\hline $\mathrm{O} 31 \mathrm{~B}-\mathrm{C} 31 \mathrm{~B}-\mathrm{C} 3 \mathrm{~B}-\mathrm{N} 2 \mathrm{~B}$ & $132(4)$ & $\mathrm{C} 3 \mathrm{~B}-\mathrm{C} 31 \mathrm{~B}-\mathrm{N} 31-\mathrm{C} 12$ & $174(2)$ \\
\hline $\mathrm{N} 31-\mathrm{C} 31 \mathrm{~B}-\mathrm{C} 3 \mathrm{~B}-\mathrm{N} 2 \mathrm{~B}$ & $-37(6)$ & $\mathrm{C} 31 \mathrm{~B}-\mathrm{N} 31-\mathrm{C} 12-\mathrm{N} 13$ & $179.8(3)$ \\
\hline $\mathrm{O} 31 \mathrm{~B}-\mathrm{C} 31 \mathrm{~B}-\mathrm{C} 3 \mathrm{~B}-\mathrm{C} 4 \mathrm{~B}$ & $-49(6)$ & $\mathrm{C} 31 \mathrm{~B}-\mathrm{N} 31-\mathrm{C} 12-\mathrm{S} 11$ & $-3.1(4)$ \\
\hline $\mathrm{N} 31-\mathrm{C} 31 \mathrm{~B}-\mathrm{C} 3 \mathrm{~B}-\mathrm{C} 4 \mathrm{~B}$ & $141(5)$ & $\mathrm{C} 17 \mathrm{~A}-\mathrm{S} 11-\mathrm{C} 12-\mathrm{N} 13$ & $0.7(2)$ \\
\hline $\mathrm{N} 2 \mathrm{~B}-\mathrm{C} 3 \mathrm{~B}-\mathrm{C} 4 \mathrm{~B}-\mathrm{C} 5 \mathrm{~B}$ & $-2(6)$ & $\mathrm{C} 17 \mathrm{~A}-\mathrm{S} 11-\mathrm{C} 12-\mathrm{N} 31$ & $-176.5(2)$ \\
\hline $\mathrm{C} 31 \mathrm{~B}-\mathrm{C} 3 \mathrm{~B}-\mathrm{C} 4 \mathrm{~B}-\mathrm{C} 5 \mathrm{~B}$ & $180(4)$ & $\mathrm{N} 31-\mathrm{C} 12-\mathrm{N} 13-\mathrm{C} 13 \mathrm{~A}$ & $178.1(2)$ \\
\hline $\mathrm{C} 3 \mathrm{~B}-\mathrm{C} 4 \mathrm{~B}-\mathrm{C} 5 \mathrm{~B}-\mathrm{O} 1 \mathrm{~B}$ & $2(5)$ & $\mathrm{S} 11-\mathrm{C} 12-\mathrm{N} 13-\mathrm{C} 13 \mathrm{~A}$ & $0.9(3)$ \\
\hline $\mathrm{C} 3 \mathrm{~B}-\mathrm{C} 4 \mathrm{~B}-\mathrm{C} 5 \mathrm{~B}-\mathrm{C} 51$ & $176(3)$ & $\mathrm{C} 12-\mathrm{N} 13-\mathrm{C} 13 \mathrm{~A}-\mathrm{C} 17 \mathrm{~A}$ & $-2.6(3)$ \\
\hline $\mathrm{N} 2 \mathrm{~B}-\mathrm{O} 1 \mathrm{~B}-\mathrm{C} 5 \mathrm{~B}-\mathrm{C} 4 \mathrm{~B}$ & $-1(3)$ & $\mathrm{C} 12-\mathrm{N} 13-\mathrm{C} 13 \mathrm{~A}-\mathrm{C} 14$ & $177.8(3)$ \\
\hline $\mathrm{N} 2 \mathrm{~B}-\mathrm{O} 1 \mathrm{~B}-\mathrm{C} 5 \mathrm{~B}-\mathrm{C} 51$ & $-176.4(18)$ & $\mathrm{C} 17 \mathrm{~A}-\mathrm{C} 13 \mathrm{~A}-\mathrm{C} 14-\mathrm{C} 15$ & $-0.6(4)$ \\
\hline $\mathrm{C} 4 \mathrm{~B}-\mathrm{C} 5 \mathrm{~B}-\mathrm{C} 51-\mathrm{C} 53$ & $155(3)$ & $\mathrm{N} 13-\mathrm{C} 13 \mathrm{~A}-\mathrm{C} 14-\mathrm{C} 15$ & $178.9(2)$ \\
\hline $\mathrm{O} 1 \mathrm{~B}-\mathrm{C} 5 \mathrm{~B}-\mathrm{C} 51-\mathrm{C} 53$ & $-31.4(18)$ & $\mathrm{C} 13 \mathrm{~A}-\mathrm{C} 14-\mathrm{C} 15-\mathrm{C} 16 \mathrm{~A}$ & $-1.6(4)$ \\
\hline $\mathrm{C} 4 \mathrm{~B}-\mathrm{C} 5 \mathrm{~B}-\mathrm{C} 51-\mathrm{C} 52$ & $-135(3)$ & $\mathrm{C} 14-\mathrm{C} 13 \mathrm{~A}-\mathrm{C} 17 \mathrm{~A}-\mathrm{C} 17$ & $2.0(4)$ \\
\hline $\mathrm{O} 1 \mathrm{~B}-\mathrm{C} 5 \mathrm{~B}-\mathrm{C} 51-\mathrm{C} 52$ & $38.5(18)$ & $\mathrm{N} 13-\mathrm{C} 13 \mathrm{~A}-\mathrm{C} 17 \mathrm{~A}-\mathrm{C} 17$ & $-177.5(2)$ \\
\hline $\mathrm{C} 5 \mathrm{~B}-\mathrm{C} 51-\mathrm{C} 52-\mathrm{C} 53$ & $-112.8(9)$ & $\mathrm{C} 14-\mathrm{C} 13 \mathrm{~A}-\mathrm{C} 17 \mathrm{~A}-\mathrm{S} 11$ & $-177.3(2)$ \\
\hline $\mathrm{C} 5 \mathrm{~B}-\mathrm{C} 51-\mathrm{C} 53-\mathrm{C} 52$ & $107.4(10)$ & $\mathrm{N} 13-\mathrm{C} 13 \mathrm{~A}-\mathrm{C} 17 \mathrm{~A}-\mathrm{S} 11$ & $3.2(3)$ \\
\hline $\mathrm{C} 5 \mathrm{~A}-\mathrm{O} 1 \mathrm{~A}-\mathrm{N} 2 \mathrm{~A}-\mathrm{C} 3 \mathrm{~A}$ & $0(3)$ & $\mathrm{C} 16 \mathrm{~A}-\mathrm{C} 17-\mathrm{C} 17 \mathrm{~A}-\mathrm{C} 13 \mathrm{~A}$ & $-1.2(4)$ \\
\hline $\mathrm{O} 1 \mathrm{~A}-\mathrm{N} 2 \mathrm{~A}-\mathrm{C} 3 \mathrm{~A}-\mathrm{C} 4 \mathrm{~A}$ & $-2(5)$ & $\mathrm{C} 16 \mathrm{~A}-\mathrm{C} 17-\mathrm{C} 17 \mathrm{~A}-\mathrm{S} 11$ & $177.9(2)$ \\
\hline $\mathrm{N} 2 \mathrm{~A}-\mathrm{C} 3 \mathrm{~A}-\mathrm{C} 4 \mathrm{~A}-\mathrm{C} 5 \mathrm{~A}$ & $3(5)$ & $\mathrm{C} 12-\mathrm{S} 11-\mathrm{C} 17 \mathrm{~A}-\mathrm{C} 13 \mathrm{~A}$ & $-2.1(2)$ \\
\hline $\mathrm{C} 3 \mathrm{~A}-\mathrm{C} 4 \mathrm{~A}-\mathrm{C} 5 \mathrm{~A}-\mathrm{O} 1 \mathrm{~A}$ & $-2(4)$ & $\mathrm{C} 12-\mathrm{S} 11-\mathrm{C} 17 \mathrm{~A}-\mathrm{C} 17$ & $178.7(3)$ \\
\hline $\mathrm{C} 3 \mathrm{~A}-\mathrm{C} 4 \mathrm{~A}-\mathrm{C} 5 \mathrm{~A}-\mathrm{C} 61$ & $178(2)$ & $\mathrm{C} 17 \mathrm{~A}-\mathrm{C} 17-\mathrm{C} 16 \mathrm{~A}-\mathrm{C} 15$ & $-1.1(4)$ \\
\hline $\mathrm{N} 2 \mathrm{~A}-\mathrm{O} 1 \mathrm{~A}-\mathrm{C} 5 \mathrm{~A}-\mathrm{C} 4 \mathrm{~A}$ & $2(2)$ & $\mathrm{C} 17 \mathrm{~A}-\mathrm{C} 17-\mathrm{C} 16 \mathrm{~A}-\mathrm{O} 16$ & $-176(3)$ \\
\hline $\mathrm{N} 2 \mathrm{~A}-\mathrm{O} 1 \mathrm{~A}-\mathrm{C} 5 \mathrm{~A}-\mathrm{C} 61$ & $-178.5(14)$ & $\mathrm{C} 14-\mathrm{C} 15-\mathrm{C} 16 \mathrm{~A}-\mathrm{C} 17$ & $2.5(5)$ \\
\hline $\mathrm{C} 4 \mathrm{~A}-\mathrm{C} 5 \mathrm{~A}-\mathrm{C} 61-\mathrm{C} 63$ & $6(3)$ & $\mathrm{C} 14-\mathrm{C} 15-\mathrm{C} 16 \mathrm{~A}-\mathrm{O} 16$ & $178(3)$ \\
\hline $\mathrm{O} 1 \mathrm{~A}-\mathrm{C} 5 \mathrm{~A}-\mathrm{C} 61-\mathrm{C} 63$ & $-173.6(9)$ & $\mathrm{C} 17-\mathrm{C} 16 \mathrm{~A}-\mathrm{O} 16-\mathrm{C} 18$ & $-12(5)$ \\
\hline $\mathrm{C} 4 \mathrm{~A}-\mathrm{C} 5 \mathrm{~A}-\mathrm{C} 61-\mathrm{C} 62$ & $-62(3)$ & $\mathrm{C} 15-\mathrm{C} 16 \mathrm{~A}-\mathrm{O} 16-\mathrm{C} 18$ & $173(3)$ \\
\hline $\mathrm{O} 1 \mathrm{~A}-\mathrm{C} 5 \mathrm{~A}-\mathrm{C} 61-\mathrm{C} 62$ & $118.1(10)$ & $\mathrm{C} 16 \mathrm{~A}-\mathrm{O} 16-\mathrm{C} 18-\mathrm{C} 18^{\mathrm{i}}$ & $165.7(9)$ \\
\hline
\end{tabular}

Symmetry codes: (i) $-x+2, y,-z+3 / 2$; (ii) $-x+2,-y+1,-z+1$.

Hydrogen-bond geometry $\left(\AA,{ }^{\circ}\right)$

\begin{tabular}{lllll}
\hline$D-\mathrm{H} \cdots A$ & $D-\mathrm{H}$ & $\mathrm{H} \cdots A$ & $D \cdots A$ & $D-\mathrm{H} \cdots A$ \\
\hline $\mathrm{N} 31-\mathrm{H} 31 \cdots \mathrm{N} 13^{\mathrm{iii}}$ & $0.82(3)$ & $2.19(3)$ & $3.003(3)$ & $173(2)$ \\
$\mathrm{C} 17-\mathrm{H} 17 \cdots \mathrm{O} 1 A^{\text {iv }}$ & 0.93 & 2.51 & $3.293(7)$ & 142 \\
$\mathrm{C} 17-\mathrm{H} 17 \cdots \mathrm{N} 2 A^{\text {iv }}$ & 0.93 & 2.55 & $3.440(19)$ & 160 \\
$\mathrm{C} 63-\mathrm{H} 63 B \cdots \mathrm{O} 31 A^{\mathrm{v}}$ & 0.97 & 2.58 & $3.440(18)$ & 148 \\
\hline
\end{tabular}

Symmetry codes: (iii) $-x+1, y,-z+1 / 2$; (iv) $x+1 / 2,-y+3 / 2, z+1 / 2$; (v) $-x+1 / 2,-y+3 / 2,-z+1$. 